\title{
Geometric inflexibility and 3-manifolds that fiber over the circle
}

\author{
J. Brock* and K. Bromberg ${ }^{\dagger}$
}

August 11, 2010

\begin{abstract}
We prove hyperbolic 3-manifolds are geometrically inflexible: a unit quasiconformal deformation of a Kleinian group extends to an equivariant bi-Lipschitz diffeomorphism between quotients whose pointwise biLipschitz constant decays exponentially in the distance form the boundary of the convex core for points in the thick part. Estimates at points in the thin part are controlled by similar estimates on the complex lengths of short curves. We use this inflexibility to give a new proof of the convergence of pseudo-Anosov double-iteration on the quasi-Fuchsian space of a closed surface, and the resulting hyperbolization theorem for closed 3-manifolds that fiber over the circle with pseudo-Anosov monodromy.
\end{abstract}

\section{Introduction}

In the study of hyperbolic structures on 3-manifolds, the rigidity theorems of Mostow and Sullivan allow for coarse methods to play a key role in the classification of structures up to isometry: it suffices to exhibit a uniformly bi-Lipschitz map between two hyperbolic 3-manifolds with the same asymptotic data to conclude they are in fact isometric.

A general theme in work of Thurston has been the notion of limiting to rigidity, wherein a family of hyperbolic structures has a quasiconformally rigid limit. Such discussions suggest a qualitative notion of inflexibility for manifolds far out in the sequence: a unit quasiconformal deformation at infinity has exponentially deteriorating effect at the basepoint as the geometry freezes around it.

This qualitative notion was made more precise for manifolds with injectivity radius bounds by McMullen (see [Mc3]), but the assumption of injectivity bounds is very restrictive. Though upper bounds on the injectivity radius in the convex core follow from tameness (now known for arbitrarily $M$ with finitely generated $\pi_{1} \mathrm{Ag}, \mathrm{CG}$ ), the lower bound is non-generic [Mc2, CCHS, CH]. In

\footnotetext{
* Research supported by the John S. Guggenheim foundation and by the NSF.

${ }^{\dagger}$ Research supported by the Alfred P. Sloan Foundation and by the NSF.
} 
this paper we prove an exponential decay theorem for the $L^{2}$-norm of a harmonic deformation a hyperbolic 3-manifold. This allows us to prove inflexibility theorems for arbitrary hyperbolic 3-manifolds. Here is a sample theorem which generalizes McMullen's result.

Theorem 1.1 (GeOMETRIC InfleXibiLity) Given a hyperbolic 3-manifold $M$, a K-bi-Lipschitz diffeomorphic hyperbolic 3-manifold $M^{\prime}$, and an $\epsilon>0$, there is a diffeomorphism $\Phi: M \rightarrow M^{\prime}$ whose bi-Lipschitz distortion in the $\epsilon$-thick part of the convex core $C(M)$ decays exponentially with the distance from $\partial C(M)$ with rate of decay depending only on $\epsilon, K$ and the topology of $\partial M$.

See Theorem 5.6 for a more precise version.

Although Theorem 1.1 does not give estimates on the bi-Lipschitz constant in the thin part, this is to be expected. Indeed, there are harmonic deformations whose distortion within a Margulis tube is roughly constant over the tube and does not decay in the depth into the tube - the pointwise bounds on the distortion (the strain) are determined by its behavior on the boundary of the tube. In this sense, Theorem 1.1 is sharp, and in fact optimal, in that we can only expect at best exponential decay of the bi-Lipschitz constant in the thick part.

On the other hand, the proof of Theorem 1.1 is quite robust and applies to a variety of other situations. For example we can control the ratio of the change in length of moderate length geodesics by constants that exponentially decay in the depth of the geodesic in the convex core. We obtain similar control over short geodesics by measuring the depth of their entire Margulis tubes. In a future paper we will apply our methods to deformations of hyperbolic cone-manifolds where the depth is measured by distance from the singular locus. For both smooth, complete hyperbolic manifolds and for cone-manifolds, the Schwarzian derivative can be similarly controlled at components of the conformal boundary that are fixed under the deformation.

We emphasize that while McMullen's inflexibility theorem is ultimately a consequence of the compactness of hyperbolic 3-manifolds with injectivity radius bounds and basepoints in the convex core, our arguments harness explicit analytic estimates on the pointwise $L^{2}$-norm of the deformation to obtain sharp estimates on the bi-Lipschitz distortion of a deformation at infinity.

Convergence results. Inflexibility provides for new approaches and techniques in the theory of Kleinian groups. To outline these results, we briefly recall notions from their deformation theory.

Given a closed surface $S$ of negative Euler-characteristic the Teichmüller space, Teich $(S)$, parametrizes pairs $(f, X)$ of marked hyperbolic surfaces

$$
f: S \rightarrow X
$$

where $f$ is a homeomorphism up to marking preserving isometry. The modular group $\operatorname{Mod}(S)$ of isotopy classes of orientation preserving self homeomorphisms of $S$ acts naturally on $\operatorname{Teich}(S)$ by $\varphi(f, X)=\left(f \circ \varphi^{-1}, X\right)$. A mapping class 
is pseudo-Anosov if for each essential isotopy class of simple closed curves $\gamma$ we have $\varphi^{n}(\gamma) \not 千 \gamma$ for $n \neq 0$.

L. Bers proved that for each pair $(X, Y) \in \operatorname{Teich}(S) \times \operatorname{Teich}(S)$ there is a unique quasi-Fuchsian simultaneous uniformization, namely, a single Kleinian group $\Gamma \cong \pi_{1}(S)$ for which $\Gamma$ leaves invariant a directed Jordan curve $\Lambda$ in $\widehat{\mathbb{C}}$ with the property that $\widehat{\mathbb{C}} \backslash \Lambda=\Omega_{X} \sqcup \Omega_{Y}$, where $\Omega_{X} / \Gamma=X$ and $\Omega_{Y} / \Gamma=Y$ (see Brs1]).

As a tool in the deformation theory of Kleinian groups, Theorem 1.1 guarantees convergence in certain cases where the depth in the convex core at the basepoint diverges quickly enough. In particular, Theorem 1.1 gives a new proof of Thurston's double limit theorem for pseudo-Anosov iteration, the main step in the hyperbolization for 3-manifolds that fiber over the circle with pseudoAnosov monodromy (see [Th2, $\mathrm{Ot}, \mathrm{Mc} 3]$ ).

Theorem 1.2 (Pseudo-Anosov Double Limits) For each $X$ and $Y$ in the Teichmüller space Teich $(S)$, and each pseudo-Anosov mapping class $\psi \in \operatorname{Mod}(S)$, the double iteration $Q\left(\psi^{-n}(X), \psi^{n}(Y)\right)$ converges algebraically and geometrically to a limit $Q_{\infty} \in A H(S)$.

See Theorem 8.3. Note that the convergence up to subsequence was proven earlier by Thurston (see [Th2). Convergence was later proven in [CT]; McMullen gave a more explicit treatment in Mc3. Note that in our result the quasi-conformal rigidity of the limit is a direct consequence of the geometric inflexibility theorem.

Because for each $n$ the manifold $Q_{n}=Q\left(\psi^{-n}(X), \psi^{n}(Y)\right)$ admits a uniformly bi-Lipschitz diffeomorphism $\Psi_{n}$ in the homotopy class of $\psi$, we may apply the inflexibility theorem to obtain an isometry $\Psi: Q_{\infty} \rightarrow Q_{\infty}$ in the homotopy class of $\psi$. The quotient $Q_{\infty} /\langle\Psi\rangle$ is a hyperbolic 3-manifold with the homotopy type of $T_{\psi}$, which is thus homeomorphic to $T_{\psi}$ by a theorem of Stallings. We arrive at Thurston's original theorem.

Theorem 1.3 (Thurston) (MAPPING Torus Hyperbolic) Let $\psi \in \operatorname{Mod}(S)$ be pseudo-Anosov. Then the mapping torus $T_{\psi}=S \times[0,1] /(x, 0) \sim(\psi(x), 1)$ admits a complete hyperbolic structure.

Curve complex distance and convex core width. To describe how Theorem 1.2 follows from Theorem 1.1, we remark that one key step is show linear growth of the width of the convex core in terms of the iterate of the pseudoAnosov applied to each factor. As the width of the core grows, the geometric effect of the next iterate decays at the basepoint exponentially fast, and convergence follows.

To show the growth in width is linear, however, the combinatorial properties of curves on surfaces play a crucial role. The collection of isotopy classes $\mathcal{S}$ of essential simple closed curves on $S$ can be encoded as a graph $\mathcal{C}(S)$ with vertices corresponding to elements of $\mathcal{S}$ and edges joining vertices if their corresponding classes can be represented by disjoint curves on $S$. This graph has the structure 
of a $\delta$-hyperbolic metric space if each edge is assigned length 1 [MM]. Though $\mathcal{C}(S)$ can be given the structure of a complex by associating $k$-simplices to $k+1$ tuples of vertices whose representatives can be realized disjointly, these higher dimensional simplices do not play a role here.

Among the many reflections of the combinatorics of $\mathcal{C}(S)$ in the geometry of hyperbolic 3-manifolds, the width of the convex core of a quasi-Fuchsian manifold is an important new example. We show the following.

Corollary 1.4 (WIDE COREs) Given a closed surface $S$, there is linear function $f$ such that the distance between the boundary components of the convex core $C(Q(X, Y))$ of a quasi-Fuchsian manifold $Q(X, Y)$ in $Q F(S)$ is bounded below by $f\left(d_{\mathcal{C}}(X, Y)\right)$.

(See Corollary 7.18$)$. Here, the distance $d_{\mathcal{C}}(X, Y)$ is shorthand: if $S$ has genus $g$, there is a uniform $L_{g}>0$ so that for each $X \in \operatorname{Teich}(S)$ has a the length of the shortest essential closed loop on $X$ is bounded by $L_{g}$. Furthermore, any two shortest loops have uniformly bounded intersection, by the collar lemma. It follows that there is a coarsely defined map from $\operatorname{Teich}(S)$ to the complex of curves, that sends each $X$ to the collection of vertices whose simple closed curves have length less than $L_{g}$ on $X$. Then $d_{\mathcal{C}}(X, Y)$ measures the maximal distance in $\mathcal{C}(S)$ between shortest curves on $X$ and on $Y$.

Since the action of pseudo-Anosov iteration has linear growth in the curve complex, it follows that the width of the convex core of the double pseudoAnosov iteration

$$
Q\left(\psi^{-n}(X), \psi^{n}(Y)\right)
$$

is linear in $n$. Combining these estimates on core width with Theorem 1.1 , Geometric Inflexibility, we obtain Thurston's original result.

It should be noted, however, that Theorem 1.2 is a convergence theorem rather than a compactness theorem. In particular, the rigidity of the limit is implicit in the proof. As such, where Thurston's original proof appealed to Sullivan's rigidity theorem after showing the limit has limit set all of $\widehat{\mathbb{C}}$, the existence of a hyperbolic structure on the mapping torus for $\psi$ here is selfcontained.

We remark that the linear growth in the width of the convex core with distance between the bounded length curves on its boundary in $\mathcal{C}(S)$ is not specific to pseudo-Anosov deformations. In particular, the methods of Theorem 1.2 extend immediately to apply to sequences $\left\{Q\left(X_{n}, Y_{n}\right)\right\}_{n}$ of quasi-Fuchsian manifolds for which we have the bounds $d_{T}\left(X_{n}, X_{n+1}\right) \leq K$ and $d_{T}\left(Y_{n}, Y_{n+1}\right) \leq K$, and the curve complex distance $d_{\mathcal{C}}\left(X_{n}, Y_{n}\right)$ grows linearly with $n$.

Ending laminations and efficient approximations. We remark that a key further application of Theorem 1.1 will be a new approach to the ending lamination conjecture $[\mathrm{BCM}]$ via efficient approximations by maximal cusps. In short, Minsky's a priori bounds theorem [Min2] guarantees that for any hyperbolic 3-manifold $M$ in the boundary of a Bers slice $B_{Y}=\{Q(X, Y): Y \in \operatorname{Teich}(S)\}$ there is an essentially canonical sequence of maximal simplices $P_{n} \in \mathcal{C}(S)$ with $P_{n} \rightarrow \lambda, \lambda \in \partial \mathcal{C}(S)$, (the boundary point $\lambda$ is the ending lamination for $M$ ) 
whose corresponding curves arise with uniformly bounded length $\ell_{M}\left(P_{n}\right)<L$ in $M$.

By an application of the grafting technique of $\mathrm{Brm} 2, \mathrm{BB}$ together with a covering argument as in BS we may, in effect, drill $P_{n}$ out of $M$ to obtain a maximal cusp $C_{n} \in \partial B_{Y}$, by a deformation that has a bounded effect on the geometry in a compact core $\mathcal{M} \subset M$. By the inflexibility theorem, the effect of this process on the geometry of $\mathcal{M}$ decays with the distance of the geodesic representatives of the curves in $P_{n}$ from $\mathcal{M}$. It follows that he sequence $C_{n}$ converges back to $M$. Since $P_{n}$ depend only on $\lambda$, it follows that $\lambda$ determines $M$. We take up this approach in BBES.

Plan of the paper. A significant component of the paper involves the study of harmonic deformations of hyperbolic 3-manifolds. In particular, estimates relating the decay of the norm of the strain field induced by a deformation to the depth in the convex core have been absent from prior treatments. The second portion of the paper develops geometric limit arguments vis a vis the complex of curves. The paper concludes with our proof of the convergence of pseudo-Anosov iteration and double-iteration on quasi-Fuchsian space, exhibiting explicitly the hyperbolic structure on the pseudo-Anosov mapping torus $T_{\psi}$.

Acknowledgements. The authors gratefully acknowledge the support of the National Science Foundation. The first author thanks Guggenheim Foundation and the second thanks the Sloan Foundation for their support. We thank MSRI for their hospitality while this work was being completed, and we thank the referee for many useful comments.

\section{Deformations}

Let $M$ be a 3-manifold and $g_{t}$ a one-parameter family of hyperbolic metrics on $M$ with $D_{t}$ the covariant derivative for the Riemannian connection for $g_{t}$. At time $t=0$ we let $g=g_{0}$ and $D=D_{0}$. We define the time zero derivative, $\eta$, of $g_{t}$ by the formula

$$
\left.\frac{d g_{t}(v, w)}{d t}\right|_{t=0}=2 g(\eta(v), w) .
$$

Then $\eta$ is a symmetric tensor of type $(1,1)$. We define the pointwise norm of $\eta$ at $p$ by choosing an orthonormal basis $\left\{e_{1}, e_{2}, e_{3}\right\}$ for $T_{p} M$ in the $g$-metric and setting

$$
\|\eta\|^{2}=\sum_{i} g\left(\eta\left(e_{i}\right), \eta\left(e_{i}\right)\right) .
$$

Note that this $L^{2}$-norm bounds the sup norm from above so that we have

$$
\|\eta(v)\| \leq\|\eta\|\|v\| .
$$

If $\eta_{t}$ is the time $t$ derivative of $g_{t}$ and $\left\|\eta_{t}\right\| \leq K$ for all $t \in[0, T]$ then by integrating we see that

$$
e^{-2 K T} g(v, v) \leq g_{T}(v, v) \leq e^{2 K T} g(v, v) .
$$


In particular, the identity map on $M$ is a $e^{K T}$-bi-Lipschitz map from $(M, g)$ to $\left(M, g_{T}\right)$.

We can also use $\eta$ to bound the change in the complex length of geodesics. Let $\gamma$ be an essential closed curve in $M$ and let $\mathcal{L}_{\gamma}(t)=\ell_{\gamma}(t)+\imath \theta_{\gamma}(t)$ be the complex length of the holonomy of $\gamma$ in the $g_{t}$-metric. The following proposition is a combination of Proposition 4.3 and Lemma 4.6 in Brm1.

Proposition 2.1 Let the harmonic strain field $\eta$ be the time zero derivative of a family of hyperbolic metrics $M_{t}=\left(M, g_{t}\right)$. Let $\gamma$ be an essential simple closed curve in $M$ and $\mathcal{L}_{\gamma}(t)=\ell_{\gamma}(t)+\imath \theta_{\gamma}(t)$ its complex length in $M_{t}$. Let $\gamma^{*}$ be the geodesic representative of $\gamma$ in $M_{0}$.

1. If the pointwise norms of $\eta$ and $D \eta$ are bounded by $K$ on $\gamma^{*}$ then

$$
\left|\mathcal{L}_{\gamma}^{\prime}(0)\right| \leq \sqrt{\frac{2}{3}} K \ell_{\gamma}(0)
$$

2. If $\gamma^{*}$ has a tubular neighborhood $U$ of radius $R$ then

$$
\int_{U}\|\eta\|^{2}+\|D \eta\|^{2} \geq\left(\frac{\left|\mathcal{L}_{\gamma}^{\prime}(t)\right|}{2 \ell_{\gamma}(t)}\right)^{2}\left(\frac{\sinh R}{\cosh R}\right)\left(2+\frac{1}{\cosh ^{2} R}\right) \text { area } \partial U .
$$

When the derivative $\eta$ is a harmonic strain field there are a number of formulas that are very useful in controlling the norm of $\eta$. Before stating these formulas we define harmonic. Given a family of hyperbolic metrics $\left(M, g_{t}\right)$ around each point we can find a one-parameter family of $\mathbb{H}^{3}$-charts $\left(U, \phi_{t}\right)$ for the hyperbolic structure induced by the $g_{t}$-metric. These charts can be viewed as a flow on a neighborhood in $\mathbb{H}^{3}$. Let $v$ be the vector field on $U$ that is the pullback of the time zero derivative of this flow. We then observe $\operatorname{sym} D v=\eta$. This follows from the fact that for vector fields $u$ and $w$ on $M$ the derivative $\left.\frac{d g_{t}(u, w)}{d t}\right|_{t=0}$ is exactly the Lie derivative of $g(u, w)$ along the vector field $v$.

The trace of $\operatorname{sym} D v$ is the divergence of $v$ and it measures the infinitesimal change in volume. The traceless part, $\operatorname{sym}_{0} D v$, is the strain of $v$ and it measures the infinitesimal change in the conformal structure. The vector field $v$ is harmonic if

$$
D^{*} D v+2 v=0 .
$$

Here $D^{*}$ is the formal adjoint of $D$. The factor of 2 arises from the fact that the Ricci curvature of a hyperbolic manifold is -2 , and the normalization guarantees that infinitesimal isometries are harmonic. We say that a strain field $\eta$ is harmonic if locally there is a divergence free and harmonic vector field $v$ with $\eta=\operatorname{sym} D v$.

Finally we note that if $\eta$ is a harmonic strain field then $* D \eta$ is also an harmonic strain field where $*$ is the Hodge star-operator (see Proposition 2.6 in $[\mathrm{HK}]$ ). While we are only really interested in controlling the size of $\eta$ we will see throughout the paper that our formulas will also involve $* D \eta$ and we will also control its size along the way. 


\section{Infinitesimal inflexibility}

The following formula is our key tool for calculating the $L^{2}$-norm of a harmonic strain field. It is Proposition 1.3 of [HK] along with the calculations on p. 36 of the same paper.

Proposition 3.1 (Hodgson-Kerkchoff) Let $M$ be a compact manifold with piecewise smooth boundary and $\eta$ a harmonic strain field. Then

$$
\int_{M}\|\eta\|^{2}+\|D \eta\|^{2}=\int_{\partial M} * D \eta \wedge \eta .
$$

The following inequality will allow us to control the boundary term in terms of point-wise bounds on the norms of $\eta$ and $D \eta$.

Lemma 3.2 We have $\|\eta\|^{2}+\|D \eta\|^{2} \geq 2\|* D \eta \wedge \eta\|$.

Proof. The inequality follows from the fact that $\|\eta-* D \eta\|^{2} \geq 0$.

The following lemma is the first step in showing that the formula from Proposition 3.1 holds on some non-compact manifolds if the strain field is bounded.

Lemma 3.3 Let $M$ be a a complete hyperbolic 3-manifold that is exhausted by compact submanifolds $M_{n}$ with the area of $\partial M_{n}$ bounded above. If $\eta$ is a harmonic strain field with the pointwise norms $\|\eta\|$ and $\|D \eta\|$ bounded above then the $L^{2}$ norm of $\eta$ and $D \eta$ is finite.

Proof. By Proposition 3.1

$$
\int_{M_{n}}\|\eta\|^{2}+\|D \eta\|^{2}=\int_{\partial M_{n}} * D \eta \wedge \eta .
$$

Since both the area of $\partial M_{n}$ and the pointwise norms of $\eta$ and $D \eta$ are bounded, Lemma 3.2 implies that the right hand side is bounded. This implies that the $L^{2}$-norm on $M$ is finite.

Let $P_{n}$ be a finite $1 / n$-net on $\partial M$. Define

$$
M(t)=\{p \in M \mid d(p, \partial M) \geq t\}
$$

and

$$
M_{n}(t)=\left\{p \in M \mid d\left(p, P_{n}\right) \geq t\right\} .
$$

Lemma 3.4 For all but an isolated set of $t>1 / n, M_{n}(t)$ is a manifold with piecewise smooth boundary.

Proof. If the boundary of $M_{n}(t)$ is not a manifold with piecewise smooth boundary then there is a geodesic of length $2 t$ in $M$ with endpoints in $P_{n}$. The set of lengths of geodesics in $M$ with endpoints in $P_{n}$ is a discrete subset of $\mathbb{R}$ so $M_{n}(t)$ must be a manifold with piecewise smooth boundary for all but an isolated set of values for $t$. 
Lemma 3.5 Let $M$ be a hyperbolic 3-manifold with piecewise smooth, compact boundary and let $\eta$ be a harmonic strain field on $M$. If $\eta$ and D $\eta$ have finite $L^{2}$-norm on $M$ then

$$
\int_{M}\|\eta\|^{2}+\|D \eta\|^{2}=\int_{\partial M} * D \eta \wedge \eta
$$

Proof. Fix a net $P_{n}$ and a $T>0$ such that the $T$-neighborhood of $P_{n}$ contains $\partial M$ and $M_{n}(T)$ is a manifold with piecewise smooth boundary. If we apply Proposition 3.1 to $M \backslash M_{n}(T)$ and rearrange terms we have

$$
\int_{M}\|\eta\|^{2}+\|D \eta\|^{2}=\int_{\partial M} * D \eta \wedge \eta-\int_{\partial M_{n}(T)} * D \eta \wedge \eta+\int_{M_{n}(T)}\|\eta\|^{2}+\|D \eta\|^{2} .
$$

By Lemma 3.4 we can choose a sequence of $t_{i} \rightarrow \infty$ such that $M_{n}\left(t_{i}\right)$ is a manifold with piecewise smooth boundary. We now apply Proposition 3.1 again to see that

$$
\int_{M_{n}(T)}\|\eta\|^{2}+\|D \eta\|^{2}=\int_{\partial M_{n}(T)} * D \eta \wedge \eta-\lim _{i \rightarrow \infty} \int_{\partial M_{n}\left(t_{i}\right)} * D \eta \wedge \eta .
$$

The function

$$
f(t)=\int_{\partial M_{n}(t)}\left(\|\eta\|^{2}+\|D \eta\|^{2}\right) d A
$$

is defined for all but a discrete set of $t$ and therefore

$$
\int_{M_{n}(T)}\|\eta\|^{2}+\|D \eta\|^{2}=\int_{T}^{\infty} f(t) d t
$$

Since the $L^{2}$-norm of $\eta$ and $D \eta$ is finite on $M_{n}(T)$ we have

$$
\lim _{t \rightarrow \infty} f(t)=0
$$

and in particular $f\left(t_{i}\right) \rightarrow 0$. Lemma 3.2 then implies that

$$
f\left(t_{i}\right) \geq 2\left|\int_{\partial M_{n}\left(t_{i}\right)} * D \eta \wedge \eta\right| .
$$

Therefore

$$
\lim _{i \rightarrow \infty} \int_{\partial M_{n}\left(t_{i}\right)} * D \eta \wedge \eta=0
$$

and

$$
\int_{M_{n}(T)}\|\eta\|^{2}+\|D \eta\|^{2}=\int_{\partial M_{n}(T)} * D \eta \wedge \eta
$$

Combining this last equality with the first equality in the proof gives us the lemma. 
Remark. Sullivan's rigidity theorem, which guarantees that a quasiconformal deformation of a finitely generated Kleinian group $\Gamma$ with support in the limit set is trivial, played a central role in Thurston's original proof of the existence of hyperbolic structures on fibered 3-manifolds. Thurston [Th1 and Bonahon [Bon1] subsequently observed that Sullivan rigidity [Sul] follows somewhat more directly if one assumes the tameness of $M=\mathbb{H}^{3} / \Gamma$, namely that $M$ is homeomorphic to the interior of a compact 3-manifold (cf. [Mc3, §3]).

Lemmas 3.3 and 3.5 give another perspective on Sullivan's result. In particular, any $\Gamma$-invariant Beltrami differential extends continuously via an averaging process to a harmonic strain field $\eta$ on $M$ with the pointwise norms of $\eta$ and $D \eta$ uniformly bounded. If $M$ is tame, then the limit set of $\Gamma$ has measure zero or is all of $\widehat{\mathbb{C}}$, by Canary's Can result that tameness implies Ahlfors' measure conjecture. In the former case, any Beltrami differential supported on the limit set is trivial. In the later case tameness also implies that $M$ is exhausted by submanifolds whose boundary has uniformly bounded area and Lemma $3.3 \mathrm{im}$ plies that the $L^{2}$-norms of $\eta$ and $D \eta$ are finite on $M$. Since $M$ has no boundary, Lemma 3.5 implies that $\eta=D \eta=0$ and the initial Beltrami differential must be trivial.

The following theorem is the key analytic estimate that underlies all of our inflexibility theorems. It should be compared with Theorem 2.15 in Mc3.

Theorem 3.6 Let $M$ be a hyperbolic 3-manifold with compact boundary and let $\eta$ be a harmonic strain field on $M$. Assume that the $L^{2}$-norms $\|\eta\|^{2}$ and $\|D \eta\|^{2}$ are finite. Then

$$
\int_{M(t)}\|\eta\|^{2}+\|D \eta\|^{2} \leq e^{-2 t} \int_{M}\|\eta\|^{2}+\|D \eta\|^{2} .
$$

Proof. We will show that

$$
\int_{M_{n}(t)}\|\eta\|^{2}+\|D \eta\|^{2} \leq e^{-2(t-2 / n)} \int_{M_{n}(2 / n)}\|\eta\|^{2}+\|D \eta\|^{2} .
$$

Taking the limit of this inequality as $n \rightarrow \infty$ will imply the theorem.

Let

$$
f(t)=\int_{M_{n}(t)}\|\eta\|^{2}+\|D \eta\|^{2} .
$$

By Lemma 3.4 we can write

$$
f(T)=\int_{T}^{\infty} \int_{\partial M_{n}(t)}\left(\|\eta\|^{2}+\|D \eta\|^{2}\right) d A d t
$$

for $T>1 / n$. Therefore

$$
\begin{aligned}
-f^{\prime}(t) & =\int_{\partial M_{n}(t)}\left(\|\eta\|^{2}+\|D \eta\|^{2}\right) d A \\
& \geq 2 \int_{\partial M_{n}(t)} * D \eta \wedge \eta \\
& \geq 2 f(t) .
\end{aligned}
$$


Integrating both sides of this inequality from $2 / n$ to infinity implies (3.1).

To go from $L^{2}$-bounds on $\eta$ to pointwise bounds we use the following mean value theorem of Hodgson and Kerckhoff. A proof can be found in Brm1.

Theorem 3.7 Let $\eta$ be a harmonic strain field on a ball $B$ of radius $R<\pi / 2$ centered at a point $p$. Then

$$
\|\eta(p)\| \leq \frac{3 \sqrt{2 \operatorname{vol} B}}{4 \pi f(R)} \sqrt{\int_{B}\|\eta\|^{2}}
$$

where $f(R)=\cosh (R) \sin (\sqrt{2} R)-\sqrt{2} \sinh (R) \cos (\sqrt{2} R)$.

We also recall the Margulis thick-thin decomposition for hyperbolic surfaces and 3-manifolds. If $M$ is a Riemannian manifold injectivity radius $\operatorname{inj}_{M}: M \rightarrow$ $\mathbb{R}^{+}$measures the radius of the maximal embedded metric ball at each point. The $\epsilon$-thin part of $M$, denoted $M^{<\epsilon}$, is the set of points $x$ in $M$ for which $\operatorname{inj}_{M}(x)<\epsilon$. Likewise the $\epsilon$-thick part $M^{\geq \epsilon}$ of $M$ is the set of $x$ for which $\operatorname{inj}_{M}(x) \geq \epsilon$.

Lemma 3.8 (Margulis) (Thick-Thin Decomposition) There exists $\varepsilon_{2}>0$ so that if $X$ is a hyperbolic surface and $\epsilon \leq \varepsilon_{2}$ then every component of $X^{<\epsilon}$ is either the open metric $R$-neighborhood of a simple closed geodesic, $R>0$, or an open horosphere modulo a discrete parabolic $\mathbb{Z}$ action.

There exists $\varepsilon_{3}>0$ so that if $M$ is a complete hyperbolic 3-manifold and $\epsilon \leq \varepsilon_{3}$ then every component of $M^{<\epsilon}$ is either the open metric tubular $R$ neighborhood $\mathbb{T}_{\epsilon}(\gamma)$ of a simple closed geodesic $\gamma$ in $M$, or an open horoball modulo a discrete parabolic $\mathbb{Z}$ or $\mathbb{Z} \oplus \mathbb{Z}$ action.

The tube $\mathbb{T}_{\epsilon}(\gamma)$ is called a Margulis tube, and the horoball quotients are called rank-1 or rank-2 cusps depending on whether the action is by a $\mathbb{Z}$ or $\mathbb{Z} \oplus \mathbb{Z}$ parabolic subgroup of $\mathrm{PSL}_{2}(\mathbb{C})$. We employ the notation $\mathbb{T}_{\varepsilon_{3}}(\gamma)=\mathbb{T}(\gamma)$.

We now apply Theorems 3.6 and 3.7 to obtain pointwise bounds on $\eta$.

Theorem 3.9 Let $M$ be a complete hyperbolic 3-manifold with compact boundary and let $\eta$ be a harmonic strain field on $M$. Then

$$
\|\eta(p)\| \leq A(\epsilon) e^{-d(p, \partial M)} \sqrt{\int_{M}\|\eta\|^{2}+\|D \eta\|^{2}}
$$

where $p \in M^{\geq \epsilon}$ and

$$
A(\epsilon)=\frac{3 e^{\epsilon} \sqrt{2 \operatorname{vol}(B)}}{4 \pi f(\epsilon)}
$$

with the function $f$ defined in Theorem 3.7 . 
Proof. Let $B$ be the ball of radius $\epsilon$ centered at $p$. Then $B$ lies in $M(d(p, \partial M)-$ $\epsilon)$. By Theorem 3.6

$$
\begin{aligned}
\int_{B}\|\eta\|^{2} & \leq \int_{B}\|\eta\|^{2}+\|D \eta\|^{2} \\
& \leq \int_{M(d(p, \partial M)-\epsilon)}\|\eta\|^{2}+\|D \eta\|^{2} \\
& \leq e^{-2(d(p, \partial M)-\epsilon)} \int_{M}\|\eta\|^{2}+\|D \eta\|^{2}
\end{aligned}
$$

We then apply Theorem 3.7 to finish the proof.

We can also control the derivative of the length of a closed geodesic.

Theorem 3.10 Let the harmonic strain field $\eta$ be the time zero derivative of a family of hyperbolic metrics $M_{t}=\left(M, g_{t}\right)$ where $M$ is a 3-manifold with compact boundary. Let $\gamma$ be an essential simple closed curve in $M$ and $\mathcal{L}_{\gamma}(t)=$ $\ell_{\gamma}(t)+\imath \theta_{\gamma}(t)$ its complex length in $M_{t}$. Let $\gamma^{*}$ be the geodesic representative of $\gamma$ in $M_{0}$.

1. If $\gamma^{*}$ is contained in $M_{0}^{\geq \epsilon}$ then

$$
\left|\mathcal{L}_{\gamma}^{\prime}(0)\right| \leq A(\epsilon) e^{-d\left(\gamma^{*}, \partial M\right)} \ell_{\gamma}(0) \sqrt{\frac{2}{3} \int_{M}\|\eta\|^{2}+\|D \eta\|^{2}}
$$

where $A(\epsilon)$ is the function given in Theorem 3.9.

2. If $\gamma^{*}$ has a tubular neighborhood $U$ of radius $R$ then

$$
\left|\mathcal{L}_{\gamma}^{\prime}(0)\right| \leq C(R) e^{-d(U, \partial M)} \ell_{\gamma}(0) \sqrt{\frac{\int_{M}\|\eta\|^{2}+\|D \eta\|^{2}}{\operatorname{area}(\partial U)}}
$$

where $1 / C(R)=2 \tanh R\left(2+\frac{1}{\cosh ^{2} R}\right)$.

Proof of (1). Applying Theorem 3.9 we see that on $\gamma^{*}$ the pointwise norm of $\eta$ and $D \eta$ is bounded by $A(\epsilon) e^{-d\left(\gamma^{*}, \partial M\right)} \sqrt{\|\eta\|^{2}+\|D \eta\|^{2}}$. We then apply (1) of Proposition 2.1 to finish the proof.

Proof of (2). By Theorem 3.6

$$
\int_{U}\|\eta\|^{2}+\|D \eta\|^{2} \leq e^{-d(U, \partial M)} \int_{M}\|\eta\|^{2}+\|D \eta\|^{2} .
$$

In this case, (2) of Proposition 2.1 finishes the proof. 


\section{Inflexibility}

There are two types of deformations of hyperbolic 3-manifolds that can be studied with our methods: quasiconformal deformations, namely, quasiconformal conjugacies of their uniformizing Kleinian groups, and cone-manifold deformations, deformations of a singular cone-manifold structure wherein the cone-angle at the cone-locus varies. In this paper we will restrict to quasiconformal deformations deformations but the two general global inflexibility theorems we prove in this section can also be applied to the study of cone-manifolds. We will carry this out in a subsequent paper.

Theorem 4.1 Let $g_{t}$ be a one-parameter family of hyperbolic metrics on a 3manifold $M$ with $t \in[a, b]$. Let $\eta_{t}$ be the time $t$ derivative of the metrics $g_{t}$ and let $N_{t}$ be a family of 3-dimensional submanifolds of $M$ such that $\eta_{t}$ is a harmonic strain field on $N_{t}$. Also assume that

$$
\sqrt{\int_{N_{t}}\left\|\eta_{t}\right\|^{2}+\left\|D_{t} \eta_{t}\right\|^{2}} \leq K
$$

for some $K>0$. Let $p$ be a point in $M$ such that for all $t \in[a, b], p$ is in $M_{t}^{\geq \epsilon}$ and

$$
d_{M_{t}}\left(p, M \backslash N_{t}\right) \geq d
$$

where $d>\epsilon$. Then

$$
\log \operatorname{bilip}\left(\Phi_{t}, p\right) \leq(t-a) K A(\epsilon) e^{-d}
$$

where $\Phi_{t}$ is the identity map from $M_{a}$ to $M_{t}$ and $A(\epsilon)$ is the function from Theorem 3.9.

Proof. Since $d>\epsilon$ the $\epsilon$-neighborhood of $p$ is contained in $N_{t}$ and is at least distance $d-\epsilon$ from $\partial N_{t}$. An application of Theorem 3.9 gives us

$$
\left\|\eta_{t}(p)\right\| \leq K A(\epsilon) e^{-d}
$$

Integrating we get

$$
\log \operatorname{bilip}\left(\Phi_{t}, p\right) \leq(t-a) K A(\epsilon) e^{-d}
$$

as desired.

Though the previous result gives no control over the bi-Lipschitz constant of the map $\Phi$ in the thin part, we may instead demonstrate exponential decay of the change in length of short curves in Margulis thin parts, which controls the geometry of the thin part itself. Here, the decay is measured in terms of the distance of the corresponding Margulis tube from the boundary. For completeness, we also bound the change in length of curves that are not short. 
Theorem 4.2 Let $g_{t}$ be a one-parameter family of hyperbolic metrics on a 3manifold $M$ with $t \in[a, b]$. Let $\eta_{t}$ be the time $t$ derivative of the metrics $g_{t}$ and let $N_{t}$ be a family of submanifolds of $M$ such that $\eta_{t}$ is a harmonic strain field on $N_{t}$. Also assume that

$$
\sqrt{\int_{N_{t}}\left\|\eta_{t}\right\|^{2}+\left\|D_{t} \eta_{t}\right\|^{2}} \leq K
$$

for some $K>0$. Let $\gamma_{t}$ be the geodesic representative on $\left(M, g_{t}\right)$ of a closed curve $\gamma$ and let $\ell_{\gamma}(t)$ be the length of $\gamma$.

1. Assume that $\gamma_{t}$ is in $M_{t}^{\geq \epsilon}$ for all $t \in[a, b]$, and that

$$
d_{M_{t}}\left(\gamma_{t}, M \backslash N_{t}\right) \geq d .
$$

Then

$$
\left|\log \frac{\ell_{\gamma}(b)}{\ell_{\gamma}(a)}\right| \leq \sqrt{2 / 3} A(\epsilon)(b-a) K e^{-d} .
$$

2. Assume $\gamma_{t}$ has a tubular neighborhood $U_{t}$ of radius at least $R$ and the area of $\partial U_{t}$ is at least B. Also assume that

$$
d_{M_{t}}\left(U_{t}, M \backslash N_{t}\right) \geq d
$$

for all $t \in[a, b]$. Then

$$
\left|\log \frac{\ell_{\gamma}(b)}{\ell_{\gamma}(a)}\right| \leq \frac{C(R)(b-a) K e^{-d}}{\sqrt{B}}
$$

where $C(R)$ is the function from Theorem 3.10 .

Proof. Both inequalities are obtained by integrating the estimates of Theorem 3.10 .

Remark. Although in the above theorem we only control the real lengths of closed geodesics it is straightforward to control their complex lengths. In particular if $\mathcal{L}_{\gamma}(t)$ is the complex length of $\gamma$ in $\left(M, g_{t}\right)$ then we can view $\imath \mathcal{L}_{\gamma}(t)$ as a point in the upper half space model of $\mathbb{H}^{2}$. Then the quantities on the right hand side of the inequalities bound the hyperbolic distance between $\imath \mathcal{L}_{\gamma}(a)$ and $\imath \mathcal{L}_{\gamma}(b)$. Note that this hyperbolic distance is an upper bound on the log of the ratio of real lengths so such a hyperbolic distance bound implies the inequalities in Theorem 4.2 .

\section{Quasiconformal deformations}

We now apply the results of the previous section to quasiconformal deformations. We begin reviewing some standard definitions. 
Let $M$ be a complete, orientable, hyperbolic 3-manifold. Its universal cover is naturally identified with $\mathbb{H}^{3}$ and $M$ may be recovered as the quotient $M=\mathbb{H}^{3} / \Gamma$ of $\mathbb{H}^{3}$ by a Kleinian group $\Gamma$, namely, a discrete subgroup of $\operatorname{Isom}^{+}\left(\mathbb{H}^{3}\right)$. The natural action of $\Gamma$ on $\widehat{\mathbb{C}}$ by Möbius transformations partitions $\widehat{\mathbb{C}}$ into its domain of discontinuity, $\Omega$, the largest subset of $\widehat{\mathbb{C}}$ where $\Gamma$ acts properly discontinuously, and its limit set $\Lambda$. Then the Kleinian manifold quotient $\left(\mathbb{H}^{3} \cup \Omega\right) / \Gamma$ is a 3 manifold with conformal boundary $\Omega / \Gamma$.

A $K$-quasiconformal deformation of a complete, orientable, hyperbolic 3manifolds $M_{0}$ is a is a map $\Psi: M_{0} \rightarrow M_{1}$ to a complete hyperbolic 3-manifold $M_{1}$ such that the lift $\tilde{\Psi}: \mathbb{H}^{3} \rightarrow \mathbb{H}^{3}$ to the universal covers extends continuously to a $K$-quasiconformal map of $\widehat{\mathbb{C}}$. If $\Psi$ is a $K$-quasiconformal deformation then it will extend to a $K$-quasiconformal map between the conformal boundaries of $M_{0}$ and $M_{1}$.

The following result is due to Reimann Rei] using work of Ahlfors Ah and Thurston Th1. For a self contained exposition see [Mc3]. It is an essential tool for the work that follows

Theorem 5.1 (Reimann) Let $\Psi: M_{0} \rightarrow M_{1}$ be a $K$-quasiconformal deformation of the complete hyperbolic 3-manifold $M_{0}$. Then there exists a oneparameter family, $M_{t}=\left(M, g_{t}\right), t \in[0,1]$, of hyperbolic 3-manifolds with time $t$ derivative $\eta_{t}$ such that the following holds:

1. The $\eta_{t}$ are harmonic strain fields and $\left\|\eta_{t}\right\|_{\infty},\left\|D_{t} \eta_{t}\right\|_{\infty} \leq 3 k$ where $k=$ $\frac{1}{2} \log K$;

2. Let $\Phi_{t}: M_{0} \rightarrow M_{t}$ be the identity map on $M$. Then $\Phi_{t}$ is $K^{\frac{3}{2}}$-bi-Lipschitz and $\Phi_{1}$ is homotopic to $\Psi$.

The convex cores $C\left(M_{t}\right)$ of the one-parameter family $M_{t}$ will play the role of $N_{t}$ when we apply Theorems 4.1 and 4.2 to $M_{t}$.

Lemma 5.2 Let $M$ be a complete hyperbolic 3-manifold such that $\pi_{1}(M)$ is finitely generated and assume that $M$ has no rank one cusps. Let $\eta$ be a harmonic strain field on $M$ such that the norms of $\eta$ and D $\eta$ are pointwise bounded by $k$. Then

$$
\int_{C(M)}\|\eta\|^{2}+\|D \eta\|^{2} \leq \operatorname{area}(\partial C(M)) k^{2} .
$$

Proof. We first replace the convex core with its $\epsilon$-neighborhood, $C_{\epsilon}(M)$. While the boundary of the convex core may not be smooth, the boundary of $C_{\epsilon}(M)$ will be $C^{1}$. We also note that area $\left(\partial C_{\epsilon}(M)\right) \rightarrow \operatorname{area}(\partial C(M))$ as $\epsilon \rightarrow 0$.

Since $\pi_{1}(M)$ is finitely generated the $M$ are both topologically and geometrically tame $([\mathrm{Ag}, \mathrm{CG}])$. In particular the convex cores $C(M)$ will be exhausted by submanifolds whose boundary has uniformly bounded area. Since the norms of $\eta$ and $D \eta$ are uniformly bounded we can apply Proposition 3.1 and Lemma3.2 to see that the $L^{2}$-norms of $\eta$ and $D \eta$ are uniformly bounded on these submanifolds which implies that the $L^{2}$-norms of $\eta$ and $D \eta$ are finite on $C_{\epsilon}(M)$. 
Applying Lemma 3.5 to $C_{\epsilon}(M)$ and taking a limit as $\epsilon \rightarrow 0$ gives us the lemma.

To make sure that objects deep in the convex core of $C\left(M_{0}\right)$ stay deep in the convex core of $C\left(M_{1}\right)$ we will use the fact that bi-Lipschitz maps of $\mathbb{H}^{3}$ take convex subsets of $\mathbb{H}^{3}$ to quasi-convex sets, a general feature of quasi-isometries between $\delta$-hyperbolic spaces. While this section only applies this observation for hyperbolic space we will later make use of this more general version in the setting of manifolds with pinched negative curvature, so we give more general form. Sometimes known as the Morse Lemma, Theorem 1.7 in Chapter of III.H of $[\mathrm{BH}]$ is one reference.

Theorem 5.3 Given constants $L>1$ and $\epsilon \in(0,1)$ there exists a $K>0$ such that the following holds. Let $X_{0}$ and $X_{1}$ be complete, simply connected Riemannian manifolds with sectional curvatures lying in $(-1-\epsilon,-1+\epsilon)$, and let $\Phi: X_{0} \rightarrow X_{1}$ be an L-bi-Lipschitz diffeomorphism. Then the $\Phi$-image of a convex set in $X_{0}$ is $K$-quasi-convex in $X_{1}$.

An example of a convex set is a geodesic - its image under a bi-Lipschitz map is an example of a quasi-geodesic. A more common way to state the above theorem is that in a space with pinched negative curvature, a quasi-geodesic is a bounded Hausdorff distance from a geodesic. In fact this is how the result is stated in $[\mathrm{BH}]$ but it is not hard to see that this implies the above theorem.

On application of the above theorem is the following proposition.

Proposition 5.4 Given $B>1$ and $\epsilon \in(0,1)$ there exists $d>0$ such that the following holds. Let $g_{0}$ and $g_{1}$ be complete Riemannian metrics on a manifold $M$ with sectional curvatures in $(-1-\epsilon,-1+\epsilon)$ and let $\phi:\left(M, g_{0}\right) \rightarrow\left(M, g_{1}\right)$ be $B$ bi-Lipschitz. Then then Hausdorff distance between $C\left(M, g_{1}\right)$ and $\phi\left(C\left(M, g_{0}\right)\right)$ is less than $d$.

Proof. For hyperbolic manifolds this is Proposition 2.16 in Mc3. It follows from Theorem 5.3 and the fact that every point in the the convex hull of a set is a uniform distance from a geodesic with endpoints in the set. Using work of Anderson And, Bowditch Bow proved this last fact for manifolds with pinched negative curvature where the uniformity constants depend on the pinching constants. Using Bowditch's work, McMullen's proof extends to the setting we have here.

The following is Corollary 2.17 in [Mc3]. The proof is a straightforward application of Proposition 5.4 .

Lemma 5.5 Let $\Phi: M_{0} \rightarrow M_{1}$ be an L-bi-Lipschitz diffeomorphism between complete hyperbolic 3-manifolds. Then there exist a constant d such that

$$
d\left(\Phi(p), M_{1} \backslash C\left(M_{1}\right)\right) \geq \frac{d\left(p, M_{0} \backslash C\left(M_{0}\right)\right)}{L}-d .
$$


We are now ready to prove our first inflexibility theorem for quasiconformal deformations.

Theorem 5.6 Let $M_{0}$ and $M_{1}$ be complete hyperbolic structures on a 3-manifold $M$ such that $M_{1}$ is a $K$-quasiconformal deformation of $M_{0}, \pi_{1}(M)$ is finitely generated, and $M_{0}$ has no rank one cusps. Then there is a bi-Lipschitz diffeomorphism

$$
\Phi: M_{0} \rightarrow M_{1}
$$

whose pointwise bi-Lipschitz constant satisfies

$$
\log \operatorname{bilip}(\Phi, p) \leq C_{1} e^{-C_{2} d\left(p, M_{0} \backslash C\left(M_{0}\right)\right)}
$$

where $p$ is in $M_{0}^{\geq \epsilon}$ and $C_{1}$ and $C_{2}$ depend only on $K, \epsilon$, and area $\left(\partial C\left(M_{0}\right)\right)$.

Proof. Let $M_{t}=\left(M, g_{t}\right)$ be the one-parameter family of hyperbolic manifolds given by Theorem 5.1 with $\eta_{t}$ the derivative of the metrics and

$$
\Phi_{t}: M_{0} \rightarrow M_{t}
$$

the given maps. By Lemma 5.2 we have

$$
\int_{C\left(M_{t}\right)}\left\|\eta_{t}\right\|^{2}+\left\|D_{t} \eta_{t}\right\|^{2} \leq \operatorname{area}\left(\partial C\left(M_{t}\right)\right) 9 k^{2}
$$

Lemma 5.5 guarantees

$$
d\left(\Phi_{t}(p), M_{t} \backslash C\left(M_{t}\right)\right) \geq \frac{d\left(p, M_{0} \backslash C\left(M_{0}\right)\right)}{K^{\frac{3}{2}}}-d .
$$

Since by Theorem 5.1 the $\Phi_{t}$ are $K^{\frac{3}{2}}$-bi-Lipschitz we have $p \in M_{t}^{\geq \epsilon^{\prime}}$ for all $t$ where $\epsilon^{\prime}=\epsilon / K^{\frac{3}{2}}$. The result then follows from Theorem 4.1 with $\Phi=\Phi_{1}$ the desired map.

For points in the thin part, the above theorem fails to yield good estimates, but this is not surprising. Indeed, one can construct examples of harmonic strain fields on Margulis tubes where the pointwise $L^{2}$-norm is roughly constant and does not decay with depth into the tube. Rather, one expects the pointwise norm of the strain at a point in a Margulis tube to depend on the depth of the boundary of the tube. Rather than pursue such a line of argument, we will bound the change in length of short geodesics where, again, the bounds will depend on the depth of the boundary of the Margulis tube not the short geodesic. Such a bound is the natural thing to expect and suffices for applications.

For completeness we also give bounds on the change in length of curves that have bounded length but are not necessarily short. We must first show that an essential curve whose geodesic representative lies deep in the convex core of $M_{0}$ also has geodesic representative in $M_{1}$ deep in the convex core. 
Proposition 5.7 Let $M_{0}=\left(M, g_{0}\right)$ and $M_{1}=\left(M, g_{1}\right)$ be hyperbolic 3-manifolds that are L-bi-Lipschitz diffeomorphic. Let $\epsilon$ a positive constant such that $L \epsilon<$ $\varepsilon_{3}$. Then there exists a constant $d=d(L, \epsilon)$ such that the following holds. Let $\gamma$ be an essential closed curve in $M$ and $\gamma_{0}$ and $\gamma_{1}$ its geodesic representatives in $M_{0}$ and $M_{1}$, respectively.

1. We have

$$
d\left(\gamma_{1}, M_{1}-C\left(M_{1}\right)\right) \geq \frac{d\left(\gamma_{0}, M_{0}-C\left(M_{0}\right)\right)}{L}-d,
$$

and,

2. if $\ell_{M_{0}}\left(\gamma_{0}\right) \leq 2 \epsilon / L$ then

$$
d\left(\mathbb{T}_{\epsilon}^{1}(\gamma), M_{1}-C\left(M_{1}\right)\right) \geq \frac{d\left(\mathbb{T}_{\epsilon}^{0}(\gamma), M_{0}-C\left(M_{0}\right)\right)}{L}-d
$$

where $\mathbb{T}^{1} \epsilon(\gamma)$ and $\mathbb{T}^{0} \epsilon(\gamma)$ denote the Margulis tubes about $\gamma$ in $M_{1}$ and $M_{0}$.

Proof. Let $\Phi: M_{0} \rightarrow M_{1}$ be the $L$-bi-Lipschitz diffeomorphism. Let $q$ be a point on $\gamma_{1}$ with

$$
d\left(q, M_{1}-C\left(M_{1}\right)\right)=d\left(\gamma_{1}, M_{1}-C\left(M_{1}\right)\right) .
$$

By Theorem 5.3, the Hausdorff distance between $\Phi\left(\gamma_{0}\right)$ and $\gamma_{1}$ is bounded by $K$ where $K$ only depends on $L$ so there exists a $q^{\prime} \in \Phi\left(\gamma_{0}\right)$ with $d\left(q, q^{\prime}\right) \leq K$. Let $p=\Phi^{-1}\left(q^{\prime}\right)$. Then

$$
d\left(p, M_{0}-C\left(M_{0}\right)\right) \geq d\left(\gamma_{0}, M_{0}-C\left(M_{0}\right)\right) .
$$

An application of Lemma 5.5 to $p$ gives us (1).

The proof of (2) is similar with one change. Again let $q$ be a point on $\partial \mathbb{T}_{\epsilon}^{1}(\gamma)$ such that

$$
d\left(q, M_{1}-C\left(M_{1}\right)\right)=d\left(\mathbb{T}_{\epsilon}^{1}(\gamma), M_{1}-C\left(M_{1}\right)\right) .
$$

The collar $\mathbb{T}_{L \epsilon}^{1}(\gamma)-\mathbb{T}_{\epsilon / L}^{1}(\gamma)$ will contain $\partial \Phi\left(\mathbb{T}_{\epsilon}^{0}(\gamma)\right)$ and the inclusion will be a homotopy equivalence since $\Phi\left(\mathbb{T}_{\epsilon}^{0}(\gamma)\right)$ is not contained in the collar. By [BM] the width of the collar is bounded above by some $W$ depending only on $\epsilon$ and $L$. Therefore there exists a $q^{\prime} \in \partial \Phi\left(\mathbb{T}_{\epsilon}^{0}(\gamma)\right)$ such that $d\left(q, q^{\prime}\right) \leq W$. The rest of the proof is the same as in (1). tions.

We can now control the length of geodesics under quasiconformal deforma-

Theorem 5.8 Let $M_{1}=\left(M, g_{1}\right)$ be a $K$-quasiconformal deformation of the hyperbolic 3-manifold $M_{0}=\left(M, g_{0}\right)$ with finitely generated fundamental group and no rank-one cusps. Let $\gamma$ be an essential simple closed curve in $M$ and $\gamma_{0}$ and $\gamma_{1}$ its geodesic representatives in $M_{0}$ and $M_{1}$ respectively. Choose $\epsilon>0$ such that $\epsilon K^{\frac{3}{2}}<\varepsilon_{3}$, and let $L>2 \epsilon>0$. Then there exists constants $C_{1}$ and $C_{2}$ depending on $K, \epsilon, L$ and area $\left(\partial C\left(M_{0}\right)\right)$ such that the following holds. 
1. If $2 \epsilon \leq \ell\left(\gamma_{0}\right) \leq L$ then

$$
\left|\log \frac{\ell\left(\gamma_{1}\right)}{\ell\left(\gamma_{0}\right)}\right| \leq C_{1} e^{-C_{2} d\left(\gamma_{0}, M_{0}-C\left(M_{0}\right)\right)} .
$$

2. If $\ell\left(\gamma_{0}\right) \leq 2 \epsilon$ then

$$
\left|\log \frac{\ell\left(\gamma_{1}\right)}{\ell\left(\gamma_{0}\right)}\right| \leq C_{1} e^{-C_{2} d\left(\mathbb{T}_{\epsilon}^{0}(\gamma), M_{0}-C\left(M_{0}\right)\right)} .
$$

Proof. As with the proof Theorem 5.6 we now only need to put together the pieces. We will use Theorem 4.2, our generic inflexibility theorem for lengths of curves. To apply this result we use the family of deformations given by Theorem 5.1 where the bound on the $L^{2}$-norms of the strain fields inside the convex core comes from Lemma 5.2. Finally, Proposition 5.7 guarantees that geodesics and tubes that are deep in the convex core stay deep in the convex core. The theorem then follows from an application of Theorem 5.6. 5.8

Remark. It is easy to see that both Theorems 5.6 and 5.8 hold for geometrically finite hyperbolic manifolds with rank-one cusps. To see this let $M_{c}^{<\delta}$ be set of points in the rank one cusps of $M$ that have injectivity radius less than $\delta$. If $M$ is geometrically finite then $C_{c}^{\delta}(M)=C(M) \backslash M_{c}^{<\delta}$ will be compact and Theorems 5.6 and 5.8 will hold if we replace $C(M)$ with $C_{c}^{\delta}(M)$. We also note that area $\left(\partial C_{c}^{\delta}(M)\right) \rightarrow \operatorname{area}(C(M))$ as $\delta \rightarrow 0$ and for all $p \in C(M)$ there exists a $\delta_{p}$ such that if $\delta<\delta_{p}$ then

$$
d\left(p, M \backslash C_{c}^{\delta}(M)\right)=d(p, M \backslash C(M)) .
$$

Therefore if we let $\delta \rightarrow 0$ we recover Theorems 5.6 and 5.8 as stated above.

In fact, the above argument applies whenever $C_{c}^{\delta}(M)$ is a manifold with compact boundary, as is the case when either the intersection of each rank one cusp with the convex core has finite volume or the entire rank one cusp is contained in the convex core.

We expect both theorems should hold for any hyperbolic 3-manifold with finitely generated fundamental group.

\section{Schwarzian derivatives}

The conformal boundary of a hyperbolic 3-manifold also has a projective structure. In this section we will obtain bounds on how this projective boundary changes during a quasiconformal deformation. We begin with some background on projective structures. One reference for this material is [Dum.

A complex projective structure on a surface $S$ can be defined in two equivalent ways. First, a complex projective structure is an atlas of charts to $\widehat{\mathbb{C}}$ whose transition functions are restrictions of Möbius transformations. Second, 
a projective structures is a developing pair $(D, \rho)$ where $D: \widetilde{S} \rightarrow \widehat{\mathbb{C}}$ is a local homeomorphism and $\rho$ is representation of $\pi_{1}(S)$ in $\mathrm{PSL}_{2} \mathbb{C}$ for which that $D \circ g(x)=\rho(g) \circ D(x)$ for all $g \in \pi_{1}(S)$ and $x \in \widetilde{S}$. The map $D$ is developing map and $\rho$ is the holonomy representation. An atlas determines a developing pair and a developing pair determines an atlas.

A projective structure determines a conformal structure on $S$ but distinct projective structures may have the same underlying conformal structure. If $X$ is a conformal structure on $S$ then we let $P(X)$ denote the space of projective structures on $S$ with conformal structure $X$.

Note that the charts that define a conformal structure on the boundary at infinity of a hyperbolic 3-manifold also define a projective structure. We refer to this projective structure as the projective boundary of the manifold. We will be interested in controlling how the projective boundary changes under a deformation fixing the conformal boundary.

The difference between two projective structures $\Sigma_{0}$ and $\Sigma_{1}$ in $P(X)$ is measured by a quadratic differential $\Phi$ determined via the Schwarzian derivative. If $f$ is the conformal map between $\Sigma_{0}$ and $\Sigma_{1}$ then the Schwarzian derivative of $f$ is the quadratic differential

$$
\Phi=\left[\left(\frac{f_{z z}}{f_{z}}\right)_{z}-\frac{1}{2}\left(\frac{f_{z z}}{f_{z}}\right)^{2}\right] d z^{2}
$$

where the derivatives are taken in projective charts for $\Sigma_{0}$ and $\Sigma_{1}$. We can then define $d\left(\Sigma_{0}, \Sigma_{1}\right)=\|\Phi\|_{\infty}$ where $\|\Phi\|_{\infty}$ is the sup-norm taken with respect to the hyperbolic metric on $X$.

There is also an infinitesimal version of the Schwarzian. If $\Sigma_{t}$ is a smooth path in $P(X)$ from $\Sigma_{0}$ to $\Sigma_{1}$ then the Schwarzian's from $\Sigma_{0}$ to $\Sigma_{t}$ determine a smooth path of quadratic differentials. The time $t$ derivative $\Phi_{t}$ of this path is also a quadratic differential. The following inequality will be useful for bounding $d\left(\Sigma_{0}, \Sigma_{1}\right)$ :

$$
\|\Phi\|_{\infty} \leq \int_{0}^{1}\left\|\Phi_{t}\right\|_{\infty} d t
$$

For each hyperbolic structure $X$ there is a unique Fuchsian projective structure $\Sigma_{F}$ in $P(X)$. For an arbitrary $\Sigma \in P(X)$ we define $\|\Sigma\|_{F}=d\left(\Sigma, \Sigma_{F}\right)$.

A key substantive difference between a conformal structure and a projective structure a projective structure carries a well defined notion of a round disk. Let $\Sigma$ be projective structure. Then a round disk on $\Sigma$ is a projective map from a round disk in $\widehat{\mathbb{C}}$ to $\Sigma$. If $M$ is a hyperbolic 3-manifold then a half-space in $M$ is a local isometry from a half space in $\mathbb{H}^{3}$ to $M$. Note that the projective boundary of a half-space in $\mathbb{H}^{3}$ is a round disk so every half space in a hyperbolic 3 -manifold extends to a round disk on the projective boundary.

The following result is our generic inflexibility theorem for Schwarzian derivatives. It should be compared to Theorems 4.1 and 4.2. In a future paper we will apply this result to hyperbolic cone-manifolds. 
Theorem 6.1 Let $g_{t}, t \in[a, b]$, be a one-parameter family of hyperbolic metrics on the interior of a 3-manifold $M$ with boundary. Let $\eta_{t}$ be the time $t$ derivative of the metrics $g_{t}$ and let $N_{t}$ be a family of submanifolds of $M$ with compact boundary such that $\eta_{t}$ is a harmonic strain field on $N_{t}$. Also assume that

$$
\sqrt{\int_{N_{t}}\left\|\eta_{t}\right\|^{2}+\left\|D_{t} \eta_{t}\right\|^{2}} \leq K
$$

for some $K>0$. Let $S$ be a component of $\partial M$ such that each hyperbolic metric $g_{t}$ extends to a fixed conformal structure $X$ on $S$ and a family of projective structures $\Sigma_{t}$ on $S$. Assume that every embedded round disk in $\Sigma_{t}$ bounds an embedded half space $H$ in $N_{t}$ and that

$$
d_{M_{t}}\left(H, M \backslash N_{t}\right) \geq d
$$

for some $d>0$. Then

$$
d\left(\Sigma_{a}, \Sigma_{b}\right) \leq C K e^{-d}
$$

where $C$ is a constant depending on $\left\|\Sigma_{a}\right\|_{F}$ and the injectivity radius of the hyperbolic metric on $X$.

Proof. Let $H$ be an embedded half space in $M_{t}$ bounding a round disk in $\Sigma_{t}$. By Theorem 3.6 we have

$$
\int_{H}\left\|\eta_{t}\right\|^{2}+\left\|D_{t} \eta_{t}\right\|^{2} \leq K^{2} e^{-2 d} .
$$

Let $\Phi_{t}$ be the holomorphic quadratic differential that is the time $t$ derivative of the family of projective structures $\Sigma_{t}$. Then by Theorem 5.5 in [Brm1] we have

$$
K^{2} e^{-2 d} \geq 2 \sqrt{\frac{2 \pi}{3}} \frac{\tanh ^{2}(\kappa / 2)}{1+2\left\|\Sigma_{t}\right\|_{F}}\left\|\Phi_{t}\right\|_{\infty}
$$

where $\kappa$ is the injectivity radius of the hyperbolic structure on $X$. Integrating this inequality finishes the proof of the theorem. For details see the proof of Theorem 1.3 in Brm1.

We will now apply this theorem to quasiconformal deformations of complete hyperbolic manifolds where some components of the conformal boundary our fixed. We will be interested in measuring the change in projective structures for these fixed components of the conformal boundary. A typical example is the deformation of a quasi-Fuchsian manifold in a Bers slice. See for example Theorem 8.2 .

Let $M$ be a complete hyperbolic 3-manifold. Then each component $X$ of the conformal boundary of $M$ will bound a component of $M \backslash C(M)$, the complement of the convex core. Label this component $\mathcal{N}(X)$ which should be thought of as a standard neighborhood of $X$ in $M$. If $X$ is a union of components of the 
projective boundary then $\mathcal{N}(X)$ is the corresponding union of components of $M \backslash C(M)$.

If $M_{t}$ is a one-parameter family of complete hyperbolic structures and $X$ is a component of conformal boundary that is fixed under the deformation then the notation $\mathcal{N}(X)$ does not distinguish which manifold the neighborhood lies in. In this situation we will use the projective structure on $X$ to label then end. Namely if $\Sigma_{t}$ is the projective boundary for $X$ in the manifold $M_{t}$ then $\mathcal{N}\left(\Sigma_{t}\right)$ is the neighborhood $\mathcal{N}(X)$ in $M_{t}$.

Theorem 5.1 gave us one-parameter family of hyperbolic manifold interpolating between the domain and range of a quasiconformal deformation. We will need to use this result again but we will also need to know that the corresponding strain fields are $L^{2}$ in a neighborhood of those ends of the boundary where the deformation is conformal. For convenience we restate Theorem 5.1 as part of the theorem below.

Theorem 6.2 Let $\Psi: M_{0} \rightarrow M_{1}$ be a K-quasiconformal deformation of the complete orientable hyperbolic 3-manifold $M_{0}$. Then there exists a one-parameter family, $M_{t}=\left(M, g_{t}\right), t \in[0,1]$, of hyperbolic metrics $g_{t}$ with time $t$ derivative $\eta_{t}$ such that the following holds:

1. The $\eta_{t}$ are harmonic strain fields and $\left\|\eta_{t}\right\|_{\infty},\left\|D_{t} \eta_{t}\right\|_{\infty} \leq 3 k$ where $k=$ $\frac{1}{2} \log K$;

2. Let $\Phi_{t}: M_{0} \rightarrow M_{t}$ be the identity map on $M$. Then $\Phi_{t}$ is $K^{\frac{3}{2}}$-bi-Lipschitz and $\Phi_{1}$ is homotopic to $\Psi$;

3. Let $X$ be a union of components of the conformal boundary $M_{0}$ such that $\Psi$ extends to a conformal map on $X$. Then $\Phi_{t}$ extends to a conformal map on $X$ for all $t$ and

$$
\int_{\mathcal{N}\left(\Phi_{t}(X)\right)}\left\|\eta_{t}\right\|^{2}+\left\|D_{t} \eta_{t}\right\|^{2}<\infty
$$

Proof. We only need to prove (3) as (1) are (2) are exactly the same as Theorem 5.1. The fact that $\Phi_{t}$ is conformal on $X$ follows directly from the construction in Rei]. To establish the $L^{2}$-bounds, we lift $\eta_{t}$ to a harmonic strain field $\tilde{\eta}_{t}$ on the universal cover $\mathbb{H}^{3}$. Then $\tilde{\eta}_{t}$ is the visual extension of a Beltrami differential $\mu_{t}$ on $\widehat{\mathbb{C}}$. By construction, $\mu_{t}$ will be zero on $\tilde{\Phi}_{t}\left(\Omega_{X}\right)$ where $\Omega_{X}$ is the component of the domain of discontinuity that descends to $X$.

Let $p$ be a point in $\mathcal{N}\left(\Phi_{t}(X)\right)$. There is a unique point $q$ in $\partial C\left(M_{t}\right)$ that is nearest to $p$. Let $\sigma$ be the shortest geodesic between $p$ and $q$, let $\tilde{\sigma}$ be a lift of this geodesic to $\mathbb{H}^{3}$ and let $\tilde{p}$ and $\tilde{q}$ be the endpoints of this geodesic which lie in the pre-images of $p$ and $q$, respectively. Let $P$ be the hyperbolic plane in $\mathbb{H}^{3}$ that contains $\tilde{q}$ and is perpendicular to $\tilde{\sigma}$. The boundary of $P$ is a circle in $\widehat{\mathbb{C}}$ that bounds a disk $D$ contained in $\tilde{\Phi}_{t}\left(\Omega_{X}\right)$. An easy calculation shows that the 
in the visual measure based at $\tilde{p}$, the ratio of the area of $D$ to the area of the the entire sphere is $\tanh d(p, q)$. This implies that

$$
\left\|\eta_{t}(p)\right\|=\left\|\tilde{\eta}_{t}(p)\right\| \leq C(1-\tanh d(p, q)) \sim 2 C e^{-2 d(p, q)}
$$

where $C$ is a constant that only depends on $\left\|\mu_{t}\right\|_{\infty}$. The area of the surface obtained by taking the locus of points in $\mathcal{N}\left(\Phi_{t}(X)\right)$ a distance $d$ from $\partial C\left(M_{t}\right)$ grows like $e^{2 d}$. Together these two estimates imply that the integral of $\left\|\eta_{t}\right\|^{2}$ over $\mathcal{N}\left(\Phi_{t}(X)\right)$ is finite.

To estimate the norm of $\left\|D_{t} \eta_{t}\right\|$ we note that the lift of this strain field is obtained by averaging $\imath \mu_{t}$ so the same argument shows that it has finite $L^{2}$ norm on $\mathcal{N}\left(\Phi_{t}(X)\right)$.

We can now prove the quasiconformal deformation version of our inflexibility theorem for Schwarzian derivatives.

Theorem 6.3 Let $\Psi: M_{0} \rightarrow M_{1}$ be a $K$-quasiconformal deformation of complete, hyperbolic 3-manifolds. Assume that the conformal boundary of $M_{0}$ is the disjoint union of two collections of components $X$ and $Y$ and that $\Psi$ extends to a conformal map on $X$. Let $\Sigma_{0}$ be the projective structure on $X$ and $\Sigma_{1}$ the projective structure on $\Psi(X)$. Let $d$ be the minimal distance between $\mathcal{N}(X)$ and $\mathcal{N}(Y)$ in $M_{0}$. Then

$$
d\left(\Sigma_{0}, \Sigma_{1}\right) \leq C_{0} e^{-C_{1} d}
$$

where $C_{0}$ and $C_{1}$ depend only on $K$, the area of the hyperbolic structure on $Y$, $\left\|\Sigma_{0}\right\|_{F}$ and the injectivity radius of the hyperbolic structure on $X$.

Proof. We want to apply Theorem 6.1 Let $M_{t}$ be the one-parameter family of hyperbolic 3-manifolds given by Theorem 6.2. Then the submanifolds $N_{t}$ will be the union of the convex cores $C\left(M_{t}\right)$ and the neighborhoods $\mathcal{N}\left(\Sigma_{t}\right)$. By Lemma 5.2 the $L^{2}$-norm of $\eta_{t}$ and $D_{t} \eta_{t}$ is finite on $C\left(M_{t}\right)$, and by (3) of Theorem 6.2 these $L^{2}$-norms are finite on $\mathcal{N}\left(\Sigma_{t}\right)$. Therefore the $L^{2}$-norms are finite on the union $N_{t}$. Just as in Lemma 5.2 the boundary of $\partial N_{t}$ will not be piecewise smooth. This can be dealt with exactly as in the proof of Lemma 5.2 and we can apply Lemma 3.5 to see that

$$
\int_{N_{t}}\left\|\eta_{t}\right\|^{2}+\left\|D_{t} \eta_{t}\right\|^{2} \leq \operatorname{area}(Y) 9 k^{2}
$$

where $\operatorname{area}(Y)$ is the area of the hyperbolic structure on $Y$.

The maps $\Phi_{t}: M_{0} \rightarrow M_{t}$ are $K^{\frac{3}{2}}$-bi-Lipschitz and such a map between hyperbolic manifolds will take a convex set to a $K_{0}$-quasi-convex set where $K_{0}$ depends on $K$. Applying this fact to $\Phi_{t}^{-1}$ we see that the Hausdorff distance between $\Phi_{t}\left(C\left(M_{0}\right)\right)$ and $C\left(M_{0}\right)$ is bounded by a constant $K_{1}$ which again only depends on $K$. In particular the distance between $\mathcal{N}\left(\Phi_{t}(X)\right)$ and $\mathcal{N}\left(\Phi_{t}(Y)\right)$ is bounded below by $d / K^{\frac{3}{2}}-K_{1}$.

Finally we see that if $D$ is round disk in $\widehat{\mathbb{C}}$ bounding a half space in $H$ then $D$ descends to an embedded disk in projective boundary of $M_{t}$ if every deck 
transformation for $M_{t}$ takes $D$ off itself. But if this is the case the same will hold for $H$ so $H$ will descend to an embedded half space in $M_{t}$.

We are now in position to apply Theorem 6.1 to see that

$$
d\left(\Sigma_{0}, \Sigma_{1}\right) \leq C_{0} e^{-C_{1} d}
$$

where $C_{0}=C$ area $(Y) 9 k^{2} e^{-K_{1}}$ with $C$ the constant from Theorem 6.1 and $C_{1}=1 / K^{\frac{3}{2}}$.

Remark. If the components of $X$ are incompressible then Nehari's Theorem Neh implies that $\left\|\Sigma_{t}\right\|_{F} \leq 3 / 2$. In particular, the constants in the previous theorem will not depend on $\left\|\Sigma_{0}\right\|_{F}$ in this case.

Remark. As with our previous inflexibility theorems for quasiconformal deformations, Theorem 6.3 also holds for certain hyperbolic 3-manifolds with rankone cusps. For example if $\left(M_{t} \cup \mathcal{N}\left(\Phi_{t}(Y)\right)\right) \backslash\left(M_{t}\right)_{c}^{\delta}$ is a compact manifold then the proof of Theorem 6.3 goes through after making the exact same modifications that were described in the remark after the proof of Theorem 5.8. Manifolds lying on the boundary of a Bers slice of a closed surface give one important case where this condition holds.

\section{Curves on surfaces and limits of surface groups}

The application of inflexibility to uniformization of 3-manifolds fibering over the circle requires us to develop some preliminary notions from algebraic and geometric convergence of Kleinian groups. We emphasize that the techniques we develop treat only the case when $S$ is closed, though many results hold more generally. We will assume $S$ is closed in the sequel.

Hyperbolic surfaces. We begin by reviewing some standard facts about hyperbolic surfaces. A proof of the following Lemma of Bers can be found in Bus.

Lemma 7.1 Given a closed surface $S$ of genus $g$ there exist positive $L_{g}$ and $L_{g}^{\prime}$ such that for any hyperbolic structure $X$ on $S$ the following holds.

1. For all points $p$ in $X$ there is an essential simple closed curve of length at most $L_{g}$ that contains $p$.

2. Any simple closed curve on $X$ of length at most $L_{g}$ can be extended to pants decomposition of total length at most $L_{g}^{\prime}$.

We will employ the thick-thin decomposition for hyperbolic surfaces as well as hyperbolic 3-manifolds from Lemma 3.8, For surfaces, the thick-part satisfies a bounded diameter condition as an application of Gauss-Bonnet.

Lemma 7.2 Each component of $X^{\geq \epsilon}$ has diameter bounded by a constant $D$ depending only on $\epsilon$ and $S$. 
A surface $X$ is $\epsilon$-thick if $X^{\geq \epsilon}=X$.

The complex of curves. Given a closed surface $S$ of negative euler characteristic, let $\mathcal{S}$ denote the collection of isotopy classes of simple closed curves on $S$. The complex of curves $\mathcal{C}(S)$, is a simplicial complex of dimension $3 g-2$ whose vertices correspond to elements of $\mathcal{S}$, and whose $k$-simplices span collections of $k+1$ vertices whose corresponding elements of $\mathcal{S}$ can be realized disjointly on $S$. Giving each simplex the standard metric, we obtain a distance function

$$
d_{\mathcal{C}}: \mathcal{S} \times \mathcal{S} \rightarrow \mathbb{N}
$$

A standard projection map from $\operatorname{Teich}(S)$ to $\mathcal{C}(S)$ is readily defined by applying the following Lemma, which is a simple application of the Collar Lemma [Bus, Thm. 4.4.6] and [MM, Lem. 2.1].

Lemma 7.3 Given $L>0$ there exists $C>0$ such that if $\alpha$ and $\beta$ are simple closed curves on $X$ of length at most $L$ then we have $d_{\mathcal{C}}(\alpha, \beta) \leq C$.

The coarse projection map

$$
\pi_{\mathcal{C}}: \operatorname{Teich}(S) \rightarrow P\left(\mathcal{C}^{0}(S)\right)
$$

of Teich $(S)$ to the set $P\left(\mathcal{C}^{0}(S)\right)$ of subsets of vertices of $\mathcal{C}(S)$, assigns to each $X \in \operatorname{Teich}(S)$ the collection of vertices of $\mathcal{C}(S)$ whose corresponding curves can be realized on $X$ with length less than $L_{g}$. By Lemma 7.1, the image $\pi_{\mathcal{C}}(X)$ is non-empty and by Lemma 7.3 it has uniformly bounded diameter, so we have a coarse notion of separation between bounded length curves on $X$ and $Y$ obtained by taking

$$
d_{\mathcal{C}}(X, Y)=\operatorname{diam}_{\mathcal{C}(S)}\left(\pi_{\mathcal{C}}(X), \pi_{\mathcal{C}}(Y)\right)
$$

Thurston's compactification. The elements of $\mathcal{S}$ naturally determine points in Thurston's compactification for Teich $(S)$, the projective measured lamination space $\mathcal{P} \mathcal{M L}(S)$. Thurston showed Teichmüller space can be compactified by the $(6 g-7)$-sphere $\mathcal{P} \mathcal{M L}(S)$ to obtain a closed ball. The action of the mapping class $\operatorname{group} \operatorname{Mod}(S)$ on $\operatorname{Teich}(S)$ extends to the compactification by homeomorphisms. Each simple closed curve $\alpha$ determines a point in $\mathcal{P} \mathcal{M L}(S)$. For further details on Thurston's construction, we point the reader to [FLP], [T], or [Bon2].

Pseudo Anosov-mapping classes. Those elements $\psi \in \operatorname{Mod}(S)$ with positive translation distance realized at a point on the interior of Teich $(S)$ are known as pseudo-Anosov mapping classes. Their action on $\mathcal{C}(S)$ is characterized by a freeness condition: for each $\gamma \in \mathcal{C}^{0}(S)$, we have $\gamma \neq \psi^{n}(\gamma)$ for any non-zero $n$. Thurston showed these elements have north-south dynamics on the compactified Teichmüller space: there is a unique stable lamination $\left[\mu^{+}\right]$and unstable lamination $\left[\mu^{-}\right]$in $\mathcal{P} \mathcal{M L}(S)$ fixed by the action of $\psi$, and for each neighborhood $U$ of $\left[\mu^{+}\right]$and each $[\gamma] \in \mathcal{P} \mathcal{M L}(S)$ with $[\gamma] \neq\left[\mu^{-}\right]$, there is an $n_{0}$ for which $\psi^{n}([\gamma])$ lies in $U$ for all $n>n_{0}$, and similarly for $\left[\mu^{-}\right]$. 
Surface groups. We discuss two related notions of convergence for hyperbolic 3 -manifolds with the homotopy type of a surface $S$. A sequence $\left\{\rho_{i}\right\}$ of discrete, faithful representations

$$
\rho_{i}: \pi_{1}(S) \rightarrow \mathrm{PSL}_{2}(\mathbb{C})
$$

converges to a limit $\rho_{\infty}$ if $\rho_{i}(\gamma) \rightarrow \rho_{\infty}(\gamma)$ in $\operatorname{PSL}_{2}(\mathbb{C})$ for every $\gamma \in \pi_{1}(S)$. The quotient topology determined by passing to conjugacy classes is the algebraic topology, and the set of all conjugacy classes of discrete, faithful representations of $\pi_{1}(S)$ to $\mathrm{PSL}_{2}(\mathbb{C})$ with this topology is denoted $A H(S)$.

On the level of quotient hyperbolic 3-manifolds one obtains a similar formulation of convergence via the notion of a marking of a hyperbolic 3-manfiold by a homotopy equivalence with $S$. Precisely, for each $i$ let $M_{i}$ be a complete hyperbolic 3-manifold and

$$
f_{i}: S \rightarrow M_{i}
$$

a homotopy equivalence. Then the marked manifolds $\left\{\left(f_{i}, M_{i}\right)\right\}$ converge to the marked manifold $\left(f_{\infty}, M_{\infty}\right)$ if there are lifts $\tilde{f}_{i}: \tilde{S} \rightarrow \tilde{M}_{i}=\mathbb{H}^{3}$ such that $\tilde{f}_{i}$ converges to $\tilde{f}_{\infty}$ uniformly on compact sets. Giving such pairs the equivalence relation

$$
(f, M) \sim(g, N)
$$

if there is an isometry $\phi: M \rightarrow N$ so that $\phi \circ f \simeq g$, the quotient topology yields the algebraic topology on the set $\{[(f, M)]\}$ of equivalence classes of marked hyperbolic 3-manifolds homotopy equivalent to $S$. The topology is equivalent to that given above for representations via the natural bijective holonomy relation between conjugacy classes of discrete faithful $\mathrm{PSL}_{2}(\mathbb{C})$ representations $\rho$ of $\pi_{1}(S)$ and equivalence classes $[(f, M)]$. We will also use $A H(S)$ to refer to the collection of equivalence classes of such marked hyperbolic 3-manifolds with the algebraic topology. When the meaning is clear from context, we will also refer to a hyperbolic 3-manifold $M$ in $A H(S)$ assuming an implicit marking by a homotopy equivalence $f: S \rightarrow M$.

As in the setting of $\operatorname{Teich}(S)$, the mapping class $\operatorname{group} \operatorname{Mod}(S)$ acts on $A H(S)$ via remarking

$$
\varphi(f, M) \mapsto\left(f \circ \varphi^{-1}, M\right) .
$$

As a result, we have the diagonal action $\varphi(Q(X, Y))=Q(\varphi(X), \varphi(Y))$ of the mapping class $\varphi \in \operatorname{Mod}(S)$ on quasi-Fuchsian space.

Geometric convergence. Let $\left(M_{n}, p_{n}\right)$ be a sequence of hyperbolic 3-manifolds with basepoint. We say that $\left(M_{n}, p_{n}\right)$ converges geometrically to a based hyperbolic 3-manifold $\left(M_{\infty}, p_{\infty}\right)$ if for every compact subset $K$ of $M_{\infty}$ containing $p_{\infty}$ and every $L>1$ there exist $L$-bilipschitz embeddings

$$
\phi_{n}:\left(K, p_{\infty}\right) \rightarrow\left(M_{n}, p_{n}\right)
$$

for $n$ sufficiently large. The maps $\phi_{n}$ are the approximating maps. We note that this form of geometric convergence is often called bi-Lipschitz convergence.

The following lemma relates geometric convergence to algebraic convergence. 
Lemma 7.4 Let $\left(M_{n}, p_{n}\right)$ converge to $\left(M_{G}, p_{G}\right)$ geometrically. Let $f: S \rightarrow M_{G}$ be a map whose image is contained in an open set $\mathcal{K}$ whose closure is compact and assume $p_{G} \in \mathcal{K}$. Let $\phi_{n}:\left(\mathcal{K}, p_{G}\right) \rightarrow\left(M_{n}, p_{n}\right)$ be approximating maps with bi-Lipschitz constant limiting to 1 , and assume that $\phi_{n} \circ f: S \rightarrow M_{n}$ are homotopy equivalences. Then $\left(\phi_{n} \circ f, M_{n}\right)$ converges to $\left(f_{\infty}, M_{\infty}\right)$ where $M_{\infty}$ is the cover of $M_{G}$ induced by the subgroup $f_{*}\left(\pi_{1}(S)\right)$ and $f_{\infty}$ is the lift of $f$.

Proof. We lift the $\phi_{n}$ to maps $\tilde{\phi}_{n}:\left(\tilde{\mathcal{K}}, \tilde{p}_{G}\right) \rightarrow\left(\mathbb{H}^{3}, \tilde{p}_{n}\right)$. Note that $\tilde{\mathcal{K}}$ is a subset of $\mathbb{H}^{3}$ and we can assume that $\tilde{p}_{G}=\tilde{p}_{n}$ and that the derivative $D \tilde{\phi}_{n}$ converges to the identity on the tangent space at $\tilde{p}_{G}$. By Arzela-Ascoli this sequence will be pre-compact in the compact-open topology and since the bi-Lipschitz constant limits to 1 , every limit will be an isometry with derivative the identity on the tangent space at $\tilde{p}_{G}$. Therefore $\tilde{\phi}_{n}$ converges to the identity map and the lemma follows.

We would like to compare an algebraic convergence to geometric convergence. We say that an algebraically convergent sequence $\left[\left(f_{n}, M_{n}\right)\right] \rightarrow\left[\left(f_{\infty}, M_{\infty}\right)\right]$ converges strongly if the following holds. Let $\left(f_{n}, M_{n}\right)$ be representatives such that $\left(f_{n}, M_{n}\right)$ converges to $\left(f_{\infty}, M_{\infty}\right)$ and let $p_{n}=f_{n}(p)$ where $p$ is a point in $S$. Let $\left(M_{G}, p_{G}\right)$ be the geometric limit of $\left(M_{n}, p_{n}\right)$. Then $\left[\left(f_{n}, M_{n}\right)\right]$ converges to $\left[\left(f_{\infty}, M_{\infty}\right)\right]$ strongly if $\left(M_{G}, p_{G}\right)=\left(M_{\infty}, p_{\infty}\right)$.

Note that if $\left(f_{n}, M_{n}\right)$ converges to $\left(M_{\infty}, p_{\infty}\right)$ and the convergence is strong then the approximating maps $\phi_{n}$ can be chosen such that if $\mathcal{K}$ is a compact set with $f_{\infty}(S) \subset \mathcal{K}$ then $f_{n}$ is homotopic to $\phi_{n} \circ f_{\infty}$.

We will use the following fundamental result of Thurston and an improvement due to R. Evans.

Theorem 7.5 (Thurston, Evans) Let $\left[\rho_{n}\right] \rightarrow[\rho]$ be a convergent sequence in $A H(S)$ and assume that for all $\alpha \in \pi_{1}(S)$, if $\rho(\alpha)$ is parabolic then $\rho_{n}(\alpha)$ is parabolic for all $n$. Then the convergence is strong.

Remark. The case when $\rho_{n}$ is assumed quasi-Fuchsian was established by Thurston (see [Th1]), and generalized by Evans ([Ev]) to setting of general manifolds in $A H(S)$. We will use exclusively the case when $\rho$ has no parabolic elements in its image; the proof in this setting is considerably easier.

\subsection{Lipschitz maps}

Let $g: X \rightarrow M$ be a 1-Lipschitz homotopy equivalence of a hyperbolic surface $X$ into a hyperbolic 3-manifold $M$. If $\alpha$ is a homotopy class of simple closed curve on $X$ then the length of the geodesic representative of $\alpha$ on $X$ bounds from above the length of its geodesic representative in $M$. As a result, geometric features of hyperbolic surfaces can be used to control the geometry of 3-manifolds (cf. [Th2, [Min1], Min2], [BCM]).

Two standard constructions of such maps are Thurston's pleated surfaces and the related simplicial hyperbolic surfaces, also introduced in [Th1] and used 
extensively by Bonahon [Bon1] and Canary [Can]. Though we will employ both constructions, we need only their consequences rather than the constructions themselves.

Theorem 7.6 (Canary) Let $S$ be a closed surface and let $M \in A H(S)$. Let $x$ and $y$ be points in the convex core of $M$. We then have a homotopy $g_{t}: X_{t} \rightarrow M$ with the following properties.

1. The family $X_{t}$ is a continuously varying family of hyperbolic metrics on $S$.

2. The maps $g_{t}$ are 1-Lipschitz.

3. The point $x$ lies in $g_{0}\left(X_{0}\right)$ and $y$ lies in $g_{1}\left(X_{1}\right)$.

In particular, for any point $x$ in the convex core of $M$, there is a 1-Lipschitz map of a hyperbolic surface into $M$ whose image contains $x$.

The previous result can be proven using simplicial hyperbolic surfaces. For the following, one needs pleated surfaces directly. We use this result only in Corollary 7.15.

Proposition 7.7 Let $\alpha^{*}$ be a closed geodesic in $M \in A H(S)$ that is homotopic to a simple closed curve $\alpha$ on $S$. Then there is a 1-Lipschitz map $X \rightarrow M$ of a hyperbolic surface $X$ that restricts to an isometry from the geodesic representative of $\alpha$ on $X$ to $\alpha^{*}$.

In this case we say that $X$ realizes $\alpha$.

The following lemma recapitulates a standard fact for pleated surfaces (see Th2]) in the setting of Lipschitz homotopy equivalences of hyperbolic surfaces and 3 manifolds. It will be useful to know this for arbitrary Lipschitz constants.

Lemma 7.8 Given $\epsilon>0$ and $B \geq 1$ there exists $\epsilon^{\prime}>0$ such that if $f: X \rightarrow M$ is a B-Lipschitz homotopy equivalence of a hyperbolic surface into a hyperbolic 3-manifold $M$ and $p$ is a point with $f(p) \in M^{<\epsilon^{\prime}}$ then we have $p \in X^{<\epsilon}$.

Proof. By Lemma 7.2, the diameter of each component of $X^{\geq \epsilon}$ is bounded by a constant $D$ that only depends on $S$ and $\epsilon$. Therefore the $f$-image of each component of $X^{\geq \epsilon}$ has diameter less than $B D$. By a theorem of Brooks and Matelski (see $[\mathrm{BM}]$ ), we may choose $\epsilon^{\prime}<\varepsilon_{3}$ small enough such that the distance between the boundaries of the $\varepsilon_{3}$-thin and the $\epsilon^{\prime}$-thin part is at least $B D$. Every component of $X^{\geq \epsilon}$ has non-abelian fundamental group while every component of $M^{<\varepsilon_{3}}$ has abelian fundamental group. Since $f$ is $\pi_{1}$-injective, the $f$-image of each component of $X^{\geq \epsilon}$ must intersect $M^{\geq \varepsilon_{3}}$ and is therefore disjoint from $M^{<\epsilon^{\prime}}$. 
Mumford's compactness theorem (see Mum) guarantees that any sequence of $\epsilon$-thick surfaces in Teich $(S)$ can be re-marked to converge in Teich $(S)$ up to subsequence; the following shows the same is true for $M_{n} \in A H(S)$ with uniformly Lipschitz markings by thick surfaces.

Proposition 7.9 Let $\epsilon>0$ be given.

1. For each sequence $\left\{X_{n}\right\}$ of $\epsilon$-thick surfaces there are markings $f_{n}: S \rightarrow X_{n}$ such that $\left(f_{n}, X_{n}\right)$ converges in $\operatorname{Teich}(S)$.

2. Let $\left(f_{n}, X_{n}\right)$ be a convergent sequence in $\operatorname{Teich}(S)$ and $g_{n}: X_{n} \rightarrow M_{n}$ $B$-Lipschitz homotopy equivalences to hyperbolic 3-manifolds $M_{n}$. Then $\left\{\left(g_{n} \circ f_{n}, M_{n}\right)\right\}$ has a convergent subsequence in $A H(S)$.

Proof. Statement (1) is a restatement of Mumford's compactness theorem for the Moduli space $\mathcal{M}(S)$ Mum. To see statement (2), note that since the sequence $\left(f_{n}, X_{n}\right)$ converges, we can place a hyperbolic metric on $S$ such that the marking maps $f_{n}$ are $B^{\prime}$-Lipschitz for some $B^{\prime}>1$. Then the maps $h_{n}=g_{n} \circ f_{n}$ are $B B^{\prime}$-Lipschitz.

Pick a point $p \in S$ and let $\tilde{p} \in \tilde{S}=\mathbb{H}^{2}$ be a point in the pre-imiage of $p$. Identifying each $\tilde{M}_{n}$ with $\mathbb{H}^{3}$, we choose lifts of $h_{n}$ such that $\tilde{h}_{n}(p)=0 \in \mathbb{H}^{3}$. Since the maps $h_{n}$ are $B B^{\prime}$-Lipschitz, it follows that for any $q \in \tilde{S}$, the set $\left\{\tilde{h}_{n}(q)\right\}$ has compact closure in $\mathbb{H}^{3}$. By the Arzela-Ascoli theorem, there exists a subsequence such that $\tilde{h}_{n}$ converges uniformly on compact sets to a map $\tilde{h}_{\infty}$ : $\tilde{S} \rightarrow \mathbb{H}^{3}$. The action of $\pi_{1}(S)$ on $\tilde{S}$ commutes with the action of a representation of $\pi_{1}(S)$ in $\mathrm{PSL}_{2}(\mathbb{C})$ so that $\tilde{h}_{\infty}$ descends to a pair $\left(h_{\infty}, M_{\infty}\right)$ where $M_{\infty}$ is the quotient 3-manifold.

\subsection{Margulis estimates}

Let $M$ be a hyperbolic manifold in $A H(S)$ and $K$ a subset of $M$. The Margulis lemma provides bounds for the number of homotopy classes of essential primitive loops of length less than $L$ that intersect $K$ such that each loop is a homotopic to a simple closed curve on $S$.

Lemma 7.10 Given $L>0$ and $D>0$ there is a $N>0$ such that the following holds. Let $M \in A H(S)$ and let $K \subset M$ be a subset of diameter at most $D$. Then the number of distinct essential homotopy classes of loops of length at most $L$ intersecting $K$ is bounded above by $N$.

Proof. By $\mathrm{BM}$ ] there exists $\epsilon>0$ such that the distance between $\partial M^{<\epsilon}$ and $\partial M \geq \varepsilon_{3}$ is at least $D+L$ where $\epsilon$ is less than the 3 -dimensional Margulis constant $\varepsilon_{3}$.

The proof then breaks into two cases. First assume that $K$ intersects $M^{<\epsilon}$. Then every loop of length at most $L$ that intersects $K$ will be contained in a 
component of $M^{<\varepsilon_{3}}$. Since $M$ lies in $A H(S), M$ has no rank-two cusps and every component of $M^{<\varepsilon_{3}}$ contains one essential primitive loop.

Now we assume that there is a point $x$ in $K \cap M^{\geq \epsilon}$. Any loop of length at most $L$ that intersects $K$ will be homotopic to a loop of length at most $L+2 D$ that intersects $x$. The number of distinct homotopy classes of loops of length at most $L+2 D$ that intersect $x$ is bounded by the quotient

$$
V=\frac{\operatorname{vol}\left(B_{\mathbb{H}^{3}}(0, L+2 D+\epsilon)\right)}{\operatorname{vol}\left(B_{\mathbb{H}^{3}}(0, \epsilon)\right)}
$$

of the volumes of balls of radius $L+2 D+\epsilon$ and $\epsilon$ about the origin in hyperbolic space $\mathbb{H}^{3}$, so taking $N=V+1$ proves the Lemma.

\subsection{Geometric limit arguments}

A subset $K \subset M$ is $\epsilon$-thick if $K \subset M^{\geq \epsilon}$.

Proposition 7.11 Let $\left(M_{n}, \omega_{n}\right)$ be hyperbolic 3-manifolds homotopy equivalent to $S$ that converge geometrically to $\left(M_{\infty}, \omega_{\infty}\right)$. Assume there exist $\epsilon>0$ and $R_{n} \rightarrow \infty$ such that the $R_{n}$-neighborhood of $\omega_{n}$ in $M_{n}$ is $\epsilon$-thick. Then $M_{\infty}$ is homotopy equivalent to $S$, and there are homtopy equivalences $f_{n}: S \rightarrow M_{n}$ and $f_{\infty}: S \rightarrow M_{\infty}$ so that $\left(f_{n}, M_{n}\right)$ converges strongly to $\left(f_{\infty}, M_{\infty}\right)$.

Proof. By Theorem 7.6 there is a hyperbolic surface $X_{n}$ and a 1-Lipschitz map $g_{n}: X_{n} \rightarrow M_{n}$ whose image contains $\omega_{n}$. Let $q_{n}$ be a point in $X_{n}$ with $g_{n}\left(q_{n}\right)=\omega_{n}$. Since $g_{n}$ is 1-Lipschitz, an $R_{n}$-neighborhood of $q_{n}$ in $X_{n}$ will be $\epsilon$-thick as well. There is a constant $K$ depending only on $\epsilon$ and the genus of $S$ such that if a hyperbolic structure $X$ on $S$ has $\epsilon$-thick neighborhood of radius at least $K$ then $X$ itself is $\epsilon$-thick. In particular for large $n$ the surfaces $X_{n}$ are themselves $\epsilon$-thick.

We now apply Proposition 7.9 to find homeomorphisms $f_{n}: S \rightarrow X_{n}$ such that $\left\{\left(f_{n}, X_{n}\right)\right\}$ converges in Teichmüller space and $\left\{\left(g_{n} \circ f_{n}, M_{n}\right)\right\}$ converges in $A H(S)$. To show the sequence converges strongly it suffices to verify it is type-preserving by an application of Theorem 7.5. After an isotopy, we can assume there is a fixed point $x \in S$ such that $f_{n}(x)=q_{n}$. Let $\alpha$ be a nontrivial loop in $S$ based at $x$. Since $\left\{\left(f_{n}, X_{n}\right)\right\}$ converges we can homotope the $f_{n}$ rel $x$ so that the loops $f_{n}(\alpha)$ have length bounded above by a constant only depending on the homotopy class of $\alpha$ rel $x$. Since each $g_{n}$ is 1-Lipschitz, the lengths of the loops $g_{n} \circ f_{n}(\alpha)$ are also uniformly bounded. If the sequence is not type-preserving there will be some $\alpha$ such that the length of the geodesic representative of $g_{n} \circ f_{n}(\alpha)$ tends to zero. In particular, for large $n$ the curve $g_{n} \circ f_{n}(\alpha)$ will be homotopic into a component of the $\epsilon$-thin part of $M_{n}$. There is then a bound on the distance from $g_{n} \circ f_{n}(\alpha)$ to this component of the thin part where the bound only depends on the length of $g_{n} \circ f_{n}(\alpha)$. But for large $n$ the $R_{n}$-neighborhood $\mathcal{N}_{R_{n}}\left(\omega_{n}\right)$ of $\omega_{n}$ has non-empty intersection with this 
component of the $\epsilon$-thin part, contradicting our assumption that $\mathcal{N}_{R_{n}}\left(\omega_{n}\right)$ lies in $M_{n}^{\geq \epsilon}$.

If follows that the sequence $\left(g_{n} \circ f_{n}, M_{n}\right)$ is type-preserving, and by Theorem 7.5 the convergence is strong. The proposition then follows.

Proposition 7.12 Given positive constants $L$ and $\epsilon$, there exist $R$ and $C$ so that the following holds. Let $M \in A H(S)$, and $\alpha$ and $\beta$ curves in $\mathcal{C}(S)$. Let $\alpha^{*}$ and $\beta^{*}$ be loops based at $\omega$ in the convex core of $M$ in the homotopy class of $\alpha$ and $\beta$, respectively, and assume that $\ell_{M}\left(\alpha^{*}\right) \leq L, \ell_{M}\left(\beta^{*}\right) \leq L$ and the neighborhood $\mathcal{N}_{R}(\omega)$ of radius $R$ about $\omega$ has injectivity radius bounded below by $\epsilon$. Then we have

$$
d_{\mathcal{C}}(\alpha, \beta) \leq C .
$$

Proof. We argue by contradiction. Assume there is a sequence $\left(M_{n}, \omega_{n}\right)$ of hyperbolic manifolds with baseframes such that $\mathcal{N}_{R_{n}}\left(\omega_{n}\right)$ is $\epsilon$-thick, $R_{n} \rightarrow \infty$, and that $\alpha_{n}$ and $\beta_{n}$ are homotopy classes in $\mathcal{C}(S)$ represented by closed loops $\alpha_{n}^{*}$ and $\beta_{n}^{*}$ in $M_{n}$ based at $\omega_{n}$ of length at most $L$ for which $d_{\mathcal{C}}\left(\alpha_{n}, \beta_{n}\right) \rightarrow \infty$.

After passing to a subsequence, $\left(M_{n}, \omega_{n}\right)$ converges geometrically to a manifold $\left(M_{\infty}, \omega_{\infty}\right)$. By Proposition 7.11, $\left(M_{\infty}, \omega_{\infty}\right)$ is homotopy equivalent to $S$, and the approximating maps are homotopy equivalences for large $n$. Choosing a compact core $K$ of $M_{\infty}$ that contains a diameter $4 L$ neighborhood of $\omega_{\infty}$, there are 2-bi-Lipschitz approximating maps $\phi_{n}: K \rightarrow M_{n}$ for large $n$ such that $\phi_{n}$ are homotopy equivalences.

The image of $K$ under $\phi_{n}$ will contain $\alpha_{n}^{*}$ and $\beta_{n}^{*}$ so $\phi_{n}^{-1}\left(\alpha_{n}^{*}\right)$ and $\phi_{n}^{-1}\left(\beta_{n}^{*}\right)$ are loops in $M_{\infty}$ of length at most $2 L$. Since $K$ is compact, there are only finitely many free homotopy classes of loops in $K$ of length at most $2 L$. This finite set of loops has finite diameter in $\mathcal{C}(S)$. Since $\phi_{n}$ is a homotopy equivalence, we conclude $d_{\mathcal{C}}\left(\alpha_{n}, \beta_{n}\right)$ is uniformly bounded, contrary to our assumption.

Given $(f, M) \in A H(S)$, and $\epsilon>0$, we let $\operatorname{short}_{\epsilon}(M)$ denote the set of isotopy classes $\alpha \in \mathcal{C}(S)$ so that $\ell_{M}(\alpha)<2 \epsilon$. It is due to Thurston, and a consequence of Lemma 7.8 and Theorem 7.6 , that there is an $\epsilon_{\mathrm{s}}>0$ so that for $\epsilon<\epsilon_{\mathrm{s}}$, a closed geodesic in $M$ of length less than $\epsilon$ lies in the homotopy class of a simple closed curve on $S$. Then for $\epsilon<\epsilon_{\mathrm{S}}$ and for each $\alpha \in \operatorname{short}_{\epsilon}(M)$ there is a component $\mathbb{T}_{\epsilon}(\alpha)$ of the $\epsilon$-thin part of $M$. We record the following immediate consequence.

Lemma 7.13 Given $(f, M) \in A H(S)$, and positive $\epsilon<\epsilon_{\mathrm{s}}$, if $M$ has no cusps, and $\operatorname{short}_{\epsilon}(M)=\emptyset$, then $M$ is $\epsilon$-thick.

Proposition 7.14 Given positive $\epsilon<\epsilon_{\mathrm{s}}$, and $R>0$ there exists $L>0$ so that the following holds. Let $f: X \rightarrow M$ be a 1-Lipschitz homotopy equivalence of a hyperbolic surface $X$ into a hyperbolic 3-manifold $M$, such that $X$ is $\epsilon$-thick. If each $\gamma \in \operatorname{short}_{\epsilon}(M)$ satisfies $\ell_{X}(\gamma)>L$ then the $R$-neighborhood $\mathcal{N}_{R}(f(X))$ is $\epsilon$-thick. 
Proof. Again we argue by contradiction and assume that we have a sequence $\left\{g_{n}: X_{n} \rightarrow M_{n}\right\}$ of 1-Lipschitz homotopy equivalences from $\epsilon$-thick surfaces $X_{n}$ with the property that the infimum of $\ell_{X_{n}}(\gamma)$ over all $\gamma \in \operatorname{short}_{\epsilon}\left(M_{n}\right)$ is at least $L_{n} \rightarrow \infty$, but the $R$-ball about $g_{n}\left(X_{n}\right)$ is not $\epsilon$-thick for any $n$.

By Proposition 7.9. there are markings $f_{n}: S \rightarrow X_{n}$ so that after passing to a subsequence $\left\{\left(f_{n}, X_{n}\right)\right\}$ converges in Teich $(S)$ and $\left\{\left(g_{n} \circ f_{n}, M_{n}\right)\right\}$ converges in $A H(S)$ to an algebraic limit $\left(g_{\infty} \circ f_{\infty}, M_{\infty}\right)$ with the property that $\operatorname{short}_{\epsilon}\left(M_{\infty}\right)=\emptyset$. Otherwise there is a $\gamma$ in $\operatorname{short}_{\epsilon}\left(M_{n}\right)$ for sufficiently large $n$, so we have

$$
\ell_{X_{n}}(\gamma) \rightarrow \infty
$$

On the other hand, convergence of $\left\{\left(f_{n}, X_{n}\right)\right\}$ in Teich $(S)$ implies $\ell_{X_{n}}(\gamma)$ converges, a contradiction.

By Theorem 7.5] the sequence $\left\{\left(g_{n} \circ f_{n}, M_{n}\right)\right\}$ converges strongly to $\left(g_{\infty} \circ\right.$ $\left.f_{\infty}, M_{\infty}\right)$ with short $_{\epsilon}\left(M_{\infty}\right)=\emptyset$; in particular, by Lemma 7.13, $M_{\infty}$ is $\epsilon$-thick. By geometric convergence, the $R$-neighborhood about $g_{n}\left(X_{n}\right)$ is $\epsilon$-thick for $n$ sufficiently large, a contradiction completing the proof.

Corollary 7.15 Given positive $R, L$ and $\epsilon<\varepsilon_{3}$ there are $C$ and $D$ so that the following holds: let $\alpha^{*}$ be a loop in a manifold $M \in A H(S)$ in the homotopy class of $\alpha \in \mathcal{C}(S)$. Assume that length of $\alpha^{*}$ is at most $L$ and that the $R$-neighborhood of $\alpha^{*}$ is not $\epsilon$-thick. Then there is a curve $\beta \in \operatorname{short}_{\epsilon}(M)$ satisfying

$$
d_{\mathcal{C}}(\alpha, \beta)<C
$$

with the property that $d_{M}(\mathbb{T}(\alpha), \mathbb{T}(\beta)) \leq D$.

Proof. If $\alpha^{*}$ is not a geodesic then it is either uniformly close to its geodesic representative or $\alpha$ is in $\operatorname{short}_{\epsilon}(M)$. In the latter case, we may take $\beta=\alpha$ and we are done. Thus we can assume that $\alpha^{*}$ is a geodesic and consider 1-Lipschitz hyperbolic surface $f: X \rightarrow M$ realizing $\alpha^{*}$.

If the surface $X$ fails to be $\epsilon$-thick itself, then the theorem follows trivially from Lemmas 7.1 and 7.8 Thus we may assume that $X$ is $\epsilon$-thick.

Applying Proposition 7.14 given $R$ there is an $L^{\prime}$ so that if the $R$-ball about $X$ fails to be $\epsilon$-thick there is a curve $\beta \in \mathcal{C}(S)$ so that $\ell_{X}(\beta)<L^{\prime}$. Since $\alpha$ has length at most $L$ on $X$, by Lemma 7.1 there is a $C$ depending on $\max \left\{L, L^{\prime}\right\}$ with the property that

$$
d_{\mathcal{C}}(\alpha, \beta)<C .
$$

Since $X$ is itself $\epsilon$-thick, there is a uniform bound depending only on $\epsilon$ and the genus of $X$ for the diameter of $X$. Hence there is a uniform bound on the distance between the geodesic representatives of $\alpha$ and $\beta$ on $X$. For any loop $\gamma$ in $M$ of length at most $\max \left\{L, L^{\prime}\right\}$ there is a bound, depending only on $\max \left\{L, L^{\prime}\right\}$, on $d_{M}(\gamma, \mathbb{T}(\gamma))$. Combining the two bounds gives the result. 7.15 
We can now prove the main theorem of this section, providing a linear lower bound on the distance between two bounded length curves in a hyperbolic manifold in terms of the distance of the curves in the curve complex. By the Margulis lemma, a short curve will have a large tubular neighborhood and therefore lie at large distance from the geodesic representatives of every other bounded length curve. In this case we will prove a stronger statement and bound the distance between the Margulis tubes. For this reason, we define $\mathbb{T}_{\epsilon}^{\prime}(\gamma)=\mathbb{T}_{\epsilon}(\gamma)$ if $\ell_{M}(\gamma)<\epsilon$ and let $\mathbb{T}_{\epsilon}^{\prime}(\gamma)$ be the geodesic representative of $\gamma$ in $M$ if $\ell_{M}(\gamma) \geq \epsilon$.

Theorem 7.16 Given $L>0$ there exist $K_{1}$ and $K_{2}$ all positive so that for $\alpha$ and $\beta$ in $\mathcal{C}^{0}(S)$, and $M \in A H(S)$, the following holds: if $\ell_{M}(\alpha)<L$ and $\ell_{M}(\beta)<L$, then

$$
d_{M}\left(\mathbb{T}_{\varepsilon_{3}}^{\prime}(\alpha), \mathbb{T}_{\varepsilon_{3}}^{\prime}(\beta)\right) \geq K_{1} d_{\mathcal{C}}(\alpha, \beta)-K_{2} .
$$

Remark. We point out that Theorem 7.16 uses in an essential way the fact that $S$ is a closed surface. If $S$ has boundary, the same statement holds if we measure distance in the pared manifold $M^{0}$ obtained by excising cusps associated to $\partial S$. All the results of the paper would then generalize in the presence of the appropriate generalization of the geometric inflexibility theorem (Theorem 5.6) to this pared setting.

Before we begin the proof of Theorem 7.16 we make a definition and prove a preliminary lemma. A $D$-coarse path in $\mathcal{C}(S)$ is a sequence of $\alpha_{i}$ in $\mathcal{C}^{0}(S)$ such that $d_{\mathcal{C}}\left(\alpha_{i}, \alpha_{i+1}\right) \leq D$.

Lemma 7.17 Given $L>0$ there exists $a D>0$ and $R>0$ such that the following holds. Let $\alpha$ and $\beta$ in $\mathcal{C}(S)$ and $M \in A H(S)$ satisfy $\ell_{M}(\alpha) \leq L$ and $\ell_{M}(\beta) \leq L$. Let $\Gamma$ be a path in $M$ from $\mathbb{T}_{\varepsilon_{3}}^{\prime}(\alpha)$ to $\mathbb{T}_{\varepsilon_{3}}^{\prime}(\beta)$. Then there are closed curves $\alpha_{i}$ with $\ell_{M}\left(\alpha_{i}\right) \leq L_{g}$ and $d_{M}\left(\alpha_{i}, \Gamma\right)<R$ such that the curves $\alpha_{i}$ describe a $D$-coarse path in $\mathcal{C}(S)$ from $\alpha$ to $\beta$.

Proof. Let $x$ be the endpoint of $\Gamma$ on $\mathbb{T}_{\varepsilon_{3}}^{\prime}(\alpha)$ and $y$ the endpoint of $\Gamma$ on $\mathbb{T}_{\varepsilon_{3}}^{\prime}(\beta)$. Let $g_{t}: Z_{t} \rightarrow M, t \in[0,1]$ be a continuous family of 1-Lipschitz maps of hyperbolic surfaces $Z_{t}$ such that $x \in g_{0}\left(Z_{0}\right)$ and $y \in g_{1}\left(Z_{1}\right)$. Such an interpolation exists by Theorem 7.6 .

There is a subinterval $[a, b] \subseteq[0,1]$ such that $x \in g_{a}\left(Z_{a}\right), y \in g_{b}\left(Z_{b}\right)$ and $g_{t}\left(Z_{t}\right) \cap \Gamma \neq \emptyset$ for all $t \in[a, b]$. Reparameterize $[a, b]$ to be the interval $[0,1]$ and replace the original homotopy with this reparametrized homotopy.

Given a simple closed curve $\gamma$ on $S$ let $U(\gamma) \subseteq[0,1]$ be the set of $t$ such that there is a simple closed curve $\gamma^{\prime}$ on $Z_{t}$, homotopic to $\gamma$, with $\gamma^{\prime} \cap g_{t}^{-1}(\Gamma) \neq \emptyset$ and $\ell_{g_{t}}\left(\gamma^{\prime}\right)<L_{g}$. By (2) of Lemma 7.1, if $U(\gamma) \cap U\left(\gamma^{\prime}\right) \neq \emptyset$ then

$$
d_{\mathcal{C}}\left(\gamma, \gamma^{\prime}\right) \leq C
$$

Let $z$ be a point in $g_{t}^{-1}(\Gamma)$. By Lemma 7.1 for each $t$ there exists $\gamma \in \mathcal{C}(S)$ such that $t \in U(\gamma)$. The open (possibly disconnected) subsets $U(\gamma)$ cover $[0,1]$ 
so we can find a collection $\alpha_{0}, \ldots, \alpha_{n}$ of distinct homotopy classes of simple closed curves in $\mathcal{C}(S)$ such that the $U\left(\alpha_{i}\right)$ satisfy

$$
U\left(\alpha_{i}\right) \cap U\left(\alpha_{i+1}\right) \neq \emptyset
$$

with $0 \in U\left(\alpha_{0}\right)$ and $1 \in U\left(\alpha_{n}\right)$. In particular the $\alpha_{i}$ are a $C$-coarse path. To finish the proof we need to show that $\alpha$ and $\beta$ are uniformly close to $\alpha_{0}$ and $\alpha_{n}$, respectively.

To see this we observe that if $\ell_{M}(\alpha)$ is sufficiently small then Lemma 7.8 guarantees that if $0 \in U(\gamma)$ we have $\gamma=\alpha$. On the other hand if $\alpha$ has a sufficiently large thick neighborhood then since $\ell_{M}(\alpha) \leq L$ Proposition 7.12 implies that if $0 \in U(\gamma)$ then $\alpha$ and $\gamma$ are uniformly close in $\mathcal{C}(S)$. If neither of these cases holds, an application of Corollary 7.15 allows us to replace $\alpha$ with a curve $\alpha^{\prime}$ that is sufficiently short so that $\alpha$ and $\alpha^{\prime}$ are uniformly close in $M$ and their corresponding vertices are uniformly close in $\mathcal{C}(S)$. We then append to $\Gamma$ a geodesic segment of length at most $R$ connecting $x$ to $\mathbb{T}_{\varepsilon_{3}}^{\prime}\left(\alpha^{\prime}\right)$ to make a new path $\Gamma^{\prime}$ and apply the previous argument to $\Gamma^{\prime}$. This process yields a coarse path $\left\{\alpha_{i}\right\}$ with $\alpha_{0}=\alpha^{\prime}$ such that the $\alpha_{i}$ have representatives in $M$ of length at most $L_{g}$ and so that each $\alpha_{i}$ intersects $\Gamma^{\prime}$.

Applying the same analysis to $\beta$ we obtain the desired coarse path.

Remark. By the Bers inequality (see [Brs2, Thm. 3], Mc1, Prop. 6.4]) given the quasi-Fuchsian manifold $Q(X, Y)$ we have $\ell_{Q(X, Y)}(\gamma) \leq 2 \ell_{X}(\gamma)$. Therefore there is a uniform $D$, depending only on $S$, such that there is a $D$-coarse path from $X$ to $Y$ in $\mathcal{C}(S)$ whose lengths in $Q(X, Y)$ are at most $L_{g}$.

Proof of 7.16. Let $\Gamma$ be the shortest geodesic from $\mathbb{T}_{\varepsilon_{3}}^{\prime}(\alpha)$ to $\mathbb{T}_{\varepsilon_{3}}^{\prime}(\beta)$ so that $d_{M}\left(\mathbb{T}_{\varepsilon_{3}}^{\prime}(\alpha), \mathbb{T}_{\varepsilon_{3}}^{\prime}(\beta)\right)=\ell(\Gamma)$. Applying Lemma 7.17 with $L$ as in the statement, we obtain a $D>0$, an $R>0$, and a $D$-coarse path $\left\{\alpha_{i}\right\}_{i=0}^{n} \subset \mathcal{C}^{0}(S)$ with $\alpha_{0}=\alpha$ and $\alpha_{n}=\beta$, with each $\alpha_{i}$ satisfying $\ell_{M}\left(\alpha_{i}\right)<L_{g}$ for $0<i<n$ and with each having distance at most $R$ from $\Gamma$. Since this path $\left\{\alpha_{i}\right\}$ is $D$-coarse we have $n \geq d_{\mathcal{C}}(\alpha, \beta) / D$.

Since $\alpha_{i}$ has length at most $L_{g}$ for $0<i<n$ and lies at distance at most $R$ from $\Gamma$, each determines a curve of length at most $2 R+L_{g}$ that intersects $\Gamma$ in the same homotopy class. The number of homotopically distinct primitive closed loops of length at most $L_{g}+2 R$ intersecting $\Gamma$ such that each is homotopic to an essential simple curve on $S$ is at least $n$, where $D(n+1) \geq d_{\mathcal{C}}(\alpha, \beta)$.

The path $\Gamma$ can be divided into $\lfloor\ell(\Gamma)\rfloor$ disjoint segments of length 1 and one segment of length at most 1 . Let $N$ be the constant given by Lemma 7.10 for the length bound $L_{g}+2 R$ and the diameter bound 1 . Then each of the $\lfloor\ell(\Gamma)\rfloor+1=\lceil\ell(\Gamma)\rceil$ segments intersects at most $N$ distinct homotopy classes of closed curves of length at most $L_{g}+2 R$ and therefore

$$
N(\ell(\Gamma)+1) \geq N\lceil\ell(\Gamma)\rceil \geq n .
$$

Combining this inequality with a lower bound on $n$ we have

$$
N(\ell(\Gamma)+1) \geq \frac{d_{\mathcal{C}}(\alpha, \beta)}{D}-1
$$


as desired.

7.16

Remark. Note that if $\alpha$ is a closed curve in $M$ of length at most $L$ then the distance between $\alpha$ and $\mathbb{T}_{\varepsilon_{3}}^{\prime}(\alpha)$ is uniformly bounded by a constant only depending on $L$. In particular Theorem 7.16 holds if we replace $\mathbb{T}_{\varepsilon_{3}}^{\prime}(\alpha)$ with any curve of length at most $L$ that is homotopic to $\alpha$.

Corollary 7.18 Given a closed surface $S$, there is linear function $f$ such that the distance between the boundary components of the convex core $C(Q(X, Y))$ of a quasifuchsian manifold $Q(X, Y)$ in $Q F(S)$ is bounded below by $f\left(d_{\mathcal{C}}(X, Y)\right)$.

Proof. Let $\alpha \in \mathcal{C}(S)$ have length on $X$ of at most the Bers constant $L_{g}$, and choose $\beta \in \mathcal{C}(S)$ similarly for $Y$. Let $\alpha^{*}$ and $\beta^{*}$ be the geodesic representatives of $\alpha$ and $\beta$, respectively, in $Q(X, Y)$. By the Bers inequality $\alpha^{*}$ and $\beta^{*}$ have length at most $2 L_{g}$. Every closed geodesic is contained in the convex core $C(Q(X, Y))$ so there are hyperbolic surfaces $Z_{\alpha}$ and $Z_{\beta}$ and 1-Lipschitz homotopy equivalences, $f_{\alpha}: Z_{\alpha} \rightarrow Q(X, Y)$ and $f_{\beta}: Z_{\beta} \rightarrow Q(X, Y)$, realizing $\alpha^{*}$ and $\beta^{*}$, respectively. These maps will have image in the convex core and each separates the two components $\partial C(Q(X, Y))$.

Let $\Gamma$ be the shortest geodesic connecting the two components of the convex core boundary. Let $x$ be a point of intersection of $\Gamma$ with the image of $Z_{\alpha}$ and $y$ a point of intersection of $\Gamma$ with the image of $Z_{\beta}$. There is a curve $\alpha_{0} \in \mathcal{C}(S)$ such that $\alpha_{0}$ has a representative on $Z_{\alpha}$ of length at most $L_{g}$ and whose image $\alpha_{0}^{*}$ intersects $x$. Note that $\alpha_{0}^{*}$ will have length at most $L_{g}$ constant and, by (2) of Lemma 7.1, there is a constant $D$ such that $d_{\mathcal{C}(S)}\left(\alpha, \alpha_{0}\right) \leq D$. Similarly, we can find a curve $\beta_{0} \in \mathcal{C}(S)$ that is represented by a loop $\beta_{0}^{*}$ that intersects $y \in Q(X, Y)$, of length at most the Bers constant and with $d_{\mathcal{C}}\left(\beta, \beta_{0}\right) \leq D$.

We want to find a lower bound for $\ell(\Gamma)$. We observe that

$$
d_{Q(X, Y)}\left(\alpha_{0}^{*}, \beta_{0}^{*}\right) \leq \ell(\Gamma)+L_{g}
$$

and that

$$
d_{\mathcal{C}(S)}(X, Y)=d_{\mathcal{C}(S)}(\alpha, \beta) \leq d_{\mathcal{C}(S)}\left(\alpha_{0}, \beta_{0}\right)+2 D .
$$

The result then follows from Theorem 7.16 .

The following Corollary controls the depth of a given curve in the convex core. We leave the proof, which follows the same lines as the above, to the reader.

Corollary 7.19 Given a closed surface $S$ and $L>0$ there is a linear function $f_{L}$ such that if $\gamma \in \mathcal{C}(S)$ and the length of $\gamma$ is at most $L$ then the distance from the geodesic representative of $\gamma$ in $Q(X, Y)$ to the boundary of the convex core is at least

$$
f_{L}\left(\min \left\{d_{\mathcal{C}}\left(X, \mathbb{T}_{\varepsilon_{3}}^{\prime}(\gamma)\right), d_{\mathcal{C}}\left(Y, \mathbb{T}_{\varepsilon_{3}}^{\prime}(\gamma)\right)\right\}\right) .
$$




\section{Pseudo-Anosov double limits}

In this section, we employ the estimates on depth in the convex core from the previous section together with the inflexibility theorems of previous sections to establish the convergence of pseudo-Anosov double iteration on quasi-Fuchsian space of a closed surface. The hyperbolization theorem for 3-manifolds that fiber over the circle follows as a consequence.

The following Theorem is a refined version of a Theorem of Masur and Minsky (see [MM, Prop. 3.6]). The proof that follows was communicated to us by M. Bestvina who attributes the argument to F. Luo. (See [BeFu, Prop. 11]).

Theorem 8.1 Let $\psi \in \operatorname{Mod}(S)$ be pseudo-Anosov with $\left[\mu^{+}\right]$and $\left[\mu^{-}\right]$the attracting and repelling laminations in $\mathcal{P} \mathcal{M L}(S)$. Then there is a $K_{\psi}$ depending only on $\psi$ so that for any $B, D>0$ the following holds.

1. There exist neighborhoods $V$ and $U$ of $\left[\mu^{+}\right]$and $\left[\mu^{-}\right]$in $\mathcal{P} \mathcal{M L}(S)$ so that for any $\alpha \in V$ and $\beta \in U$ we have

$$
d_{\mathcal{C}}\left(\alpha, \psi^{n}(\beta)\right) \geq K_{\psi} n+B .
$$

2. There exists a subset $W$ in $\mathcal{P} \mathcal{M L}(S) \backslash(U \cup V)$ such that any path in $\mathcal{C}(S)$ from a curve in $U$ to a curve in $V$ contains a subpath of length at least $D$.

3. For each $\alpha \in U, \beta \in V$ and $\gamma \in W$ we have

$$
d_{\mathcal{C}}\left(\gamma, \psi^{-n}(\alpha)\right) \geq K_{\psi} n+B \quad \text { and } \quad d_{\mathcal{C}}\left(\gamma, \psi^{n}(\beta)\right) \geq K_{\psi} n+B .
$$

4. Furthermore, given any curve $\beta$, the sets $U, V$, and $W$ may be taken so that any one of them contains $\beta$.

Proof. Let $V^{\prime}$ and $U^{\prime}$ be neighborhoods of $\left[\mu^{+}\right]$and $\left[\mu^{-}\right]$in $\mathcal{P} \mathcal{M L}(S)$ such that for simple closed curves $\alpha \in V^{\prime}$ and $\beta^{\prime} \in U^{\prime}$ the intersection of $\alpha$ and $\beta$ is non-empty. Then $V^{\prime}$ and $U^{\prime}$ are necessarily disjoint, and we let

$$
W^{\prime}=\mathcal{P} \mathcal{M L}(S) \backslash\left(V^{\prime} \cup U^{\prime}\right)
$$

be their complement in $\mathcal{P} \mathcal{M L}(S)$. Then the north-south dynamics of $\psi$ guarantees that for any compact subset $\mathcal{K}$ of $\mathcal{P} \mathcal{M L}(S)$ that does not contain $\left[\mu^{+}\right]$ we have $\psi^{i}\left(U^{\prime}\right)$ contains $\mathcal{K}$ for some positive $i$. In particular, since $W^{\prime} \cup V^{\prime}$ is compact there is an $N$ so that we have $\psi^{N}\left(W^{\prime} \cup V^{\prime}\right) \subset V^{\prime}$.

We claim that for any curves $\alpha \in \psi^{m N}\left(V^{\prime}\right)$ and $\beta \in U^{\prime}$ we have

$$
d_{\mathcal{C}}(\alpha, \beta) \geq m+1 .
$$

We first note that if $\alpha \in \psi^{i}\left(V^{\prime}\right)$ and $\beta \in \psi^{i}\left(U^{\prime}\right)$ then any geodesic in the curve complex connecting them will contain a curve in $\psi^{i}\left(W^{\prime}\right)$, the complement 
of the two sets, and the distance between $\alpha$ and $\beta$ will be greater than 2 . We also note that $\psi^{(m+1) N}\left(W^{\prime}\right) \subset \psi^{m N}\left(V^{\prime}\right)$.

We prove the inequality by induction. By the observation above, the inequality (8.4) is true when $m=1$. To complete the induction, note that we have $\psi^{i}\left(U^{\prime}\right) \subset \psi^{i+N}\left(U^{\prime}\right)$. Therefore $U^{\prime}$ lies in $\psi^{(m+1) N}\left(U^{\prime}\right)$ and $\beta$ is in $\psi^{(m+1) N}\left(U^{\prime}\right)$. Any curve complex geodesic connecting $\beta$ to $\alpha$ will therefore contain a curve $\gamma$ in $\psi^{(m+1) N}\left(W^{\prime}\right)$. Since $\psi^{(m+1) N}\left(W^{\prime}\right)$ is contained in $\psi^{m N}\left(V^{\prime}\right)$ the curve $\gamma$ is contained in $\psi^{m N}\left(V^{\prime}\right)$, and we have $d_{\mathcal{C}}(\beta, \gamma) \geq m+1$ by induction. But since $\gamma$ lies on a geodesic joining $\alpha$ to $\beta$, we have

$$
\begin{aligned}
d_{\mathcal{C}}(\alpha, \beta) & =d_{\mathcal{C}}(\alpha, \gamma)+d_{\mathcal{C}}(\gamma, \beta) \\
& \geq m+2
\end{aligned}
$$

completing the induction.

Let $V_{0}=\cup_{i=0}^{N-1} \psi^{i}\left(V^{\prime}\right)$. There is an $M>0$ such that $\psi^{M N}\left(V_{0}\right) \subset V^{\prime}$. Let $n$ be an integer greater than $M$ and assume $k$ is a non-negative integer less than $N$. We then observe that

$$
\psi^{n N+k}\left(V^{\prime}\right) \subset \psi^{n N}\left(V_{0}\right) \subset \psi^{n-M}\left(V^{\prime}\right)
$$

and therefore

$$
d_{\mathcal{C}}(\alpha, \beta) \geq n-M+1
$$

for any $\alpha \in \psi^{n N+k}\left(V^{\prime}\right)$ and $\beta \in U^{\prime}$. Alternatively if $\alpha \in \psi^{n}\left(V^{\prime}\right)$ and $\beta \in U^{\prime}$ then

$$
d_{\mathcal{C}}(\alpha, \beta) \geq\left\lfloor\frac{n}{N}\right\rfloor-M+1 \geq \frac{n}{N}-M .
$$

We now set $V^{\prime \prime}=\psi^{(B+M) N}\left(V^{\prime}\right)$. Any $\alpha \in V^{\prime \prime}$ has image $\psi^{n}(\alpha)$ lying in $\psi^{(B+M) N+n}\left(V^{\prime}\right)$ so we have

$$
d_{\mathcal{C}}\left(\psi^{n}(\alpha), \beta\right) \geq \frac{(B+M) N+n}{N}-M=\frac{n}{N}+B
$$

and $U^{\prime}$ and $V^{\prime \prime}$ satisfy (1).

We may assume that $B>D$ and let $W=\mathcal{P} \mathcal{M L}(S) \backslash\left(U^{\prime} \cup V^{\prime \prime}\right)$ so that any path from $U^{\prime}$ to $V^{\prime \prime}$ contains a subpath of length at least $D$. Let $N^{\prime}=$ $(B+M+1) N$ and let $U=\psi^{-N^{\prime}}\left(U^{\prime}\right)$ and $V=\psi^{N^{\prime}}\left(V^{\prime \prime}\right)$. Since $U \subset U^{\prime}$ and $V \subset V^{\prime \prime}(2)$ will still hold $U, V$ and $W$. We also note that the sets $U$ and $\psi^{-2 N^{\prime}}(V)$ will satisfy $(1)$ and $W$ is contained in $\psi^{-2 N^{\prime}}(V)$. A similar statement holds for $\psi^{2 N^{\prime}}(U)$ and $V$ with $W$ contained in $\psi^{2 N^{\prime}}(V)$. Therefore (3) will hold.

For (4) we note that we can replace $U, V$ and $W$ with $\psi^{n}(U), \psi^{n}(V)$ and $\psi^{n}(W)$ for any integer $n$. We also note that

$$
\bigcup_{n \in \mathbb{Z}} \psi^{n}(V)=\mathcal{P} \mathcal{M L}(S) \backslash\left[\mu^{+}\right] \quad \text { and } \quad \bigcup_{n \in \mathbb{Z}} \psi^{n}(U)=\mathcal{P} \mathcal{M L}(S) \backslash\left[\mu^{-}\right]
$$

and that

$$
\begin{aligned}
\bigcup_{n \in \mathbb{Z}} \psi^{n}(W) & =\mathcal{P} \mathcal{M L}(S) \backslash\left(\bigcap_{n \in \mathbb{Z}} \psi^{n}(V) \cup \bigcap_{n \in \mathbb{Z}} \psi^{n}(U)\right) \\
& =\mathcal{P} \mathcal{M L}(S) \backslash\left\{\left[\mu^{+}\right],\left[\mu^{-}\right]\right\} .
\end{aligned}
$$


If we want a fixed curve $\beta$ to be in $U$ we choose $n$ large enough such that $\beta$ is in $\psi^{n}(U)$ and then replace $U$ with $\psi^{n}(U), V$ with $\psi^{n}(V)$ and $W$ with $\psi^{n}(W)$.

\subsection{Convergence of iteration}

Let $S$ be a closed surface. Given $Y \in \operatorname{Teich}(S)$, the Bers slice

$$
B_{Y}=\{Q(X, Y): X \in \operatorname{Teich}(S)\} \subset A H(S)
$$

parametrizes Teich $(S)$ by quasi-Fuchsian manifolds. Since the Bers slice $B_{Y}$ has compact closure in $A H(S)$ Brs2], the sequence $\left\{Q\left(\psi^{-n}(X), Y\right)\right\}_{n}$ will have a convergent subsequence. It was established in Mc3 via a geometric limit argument that the sequence converges. We give a new proof as an example of our methods.

Theorem 8.2 Let $\psi \in \operatorname{Mod}(S)$ be a pseudo-Anosov mapping class. Then the iteration $\left\{Q\left(\psi^{n}(X), Y\right)\right\}_{n}$ converges in $A H(S)$.

Proof. Let $T_{n}$ be the distance between the components of the boundary of convex cores of $Q\left(\psi^{n} X, Y\right)$. Choose closed geodesics $\alpha$ on $X$ and $\beta$ on $Y$ of length less than the Bers constant $L_{g}$ for $S$. Then applying Theorem 8.1 there is a positive integer $N$, so that

$$
d_{\mathcal{C}}\left(\psi^{n+N}(\alpha), \beta\right) \geq K n .
$$

Since $\psi^{n+N}(\alpha)$ and $\beta$ have lengths on $\psi^{n+N}(X)$ and $Y$ (respectively) bounded by $L_{g}$, we have

$$
d_{\mathcal{C}}\left(\psi^{n+N}(X), Y\right)=d_{\mathcal{C}}\left(\psi^{n+N}(\alpha), \beta\right) \geq K n .
$$

Applying Corollary 7.18 , we have

$$
T_{n}>K_{1} n-K_{2} .
$$

The pseudo-Anosov mapping class $\psi$ is uniformly quasiconformal as a mapping from $\psi^{n}(X)$ to $\psi^{n+1}(X)$ (independent of $n$ ). Let $d_{n}$ be the distance between $Q\left(\psi^{n} X, Y\right)$ and $Q\left(\psi^{n+1} X, Y\right)$ in the Bers slice $B_{Y}$ as in Theorem 6.3, Then there are constants $C_{1}$ and $C_{2}$ such that

$$
\begin{aligned}
d_{n} & \leq C_{1} e^{-C_{2} T_{n}} \\
& \leq C_{1} e^{-C_{2}\left(K_{1} n-K_{2}\right)} .
\end{aligned}
$$

This implies that $Q\left(\psi^{n}(X), Y\right)$ is a Cauchy sequence and hence convergent. 8. . 


\subsection{Double Limits}

We now examine the pseudo-Anosov double iteration

$$
Q_{n}=Q\left(\psi^{-n}(X), \psi^{n}(Y)\right)
$$

Thurston's double limit theorem [Th2] guarantees that the sequence has a convergent subsequence. In CT a proof of convergence is outlined that uses the Mostow rigidity theorem - in [Mc3], McMullen showed convergence explicitly.

Using the geometric inflexibility theorem proven here, we will give a single unified proof of this and other convergence convergence results that is independent of Thurston's double limit theorem.

Theorem 8.3 (Pseudo-Anosov Double Limits) Given $X, Y$ in Teich $(S)$, and a pseudo-Anosov mapping class $\psi \in \operatorname{Mod}(S)$, the double iteration $\left\{Q_{n}\right\}_{n}$ converges strongly in $A H(S)$.

For each $n$ there is a $K$-quasi-conformal deformation from $Q_{n}$ to $Q_{n+1}$ where $K$ bounds the Teichmüller distance from $X$ to $\psi^{-1}(X)$ and from $Y$ to $\psi(Y)$. Let $\phi_{n}: Q_{n} \rightarrow Q_{n+1}$ be the map given by Theorem 5.6. We note that in the application of the inflexibility theorems, the constants that arise in Theorem 5.6 and 5.8 depend on $K$ but not on $n$.

We begin with a criterion to ensure that a curve $\gamma$ has a convergent sequence of geodeisc lengths $\ell_{Q_{n}}(\gamma)$.

Proposition 8.4 Given $L>0$ there exists $B>0$ so that the following holds: if given $\gamma \in \mathcal{C}^{0}(S)$ there exists $N \in \mathbb{N}$ for which $\ell_{Q_{N}}(\gamma)<L$ and for all $n$ we have

$$
\min \left\{d_{\mathcal{C}}\left(\psi^{N+n}(Y), \gamma\right), d_{\mathcal{C}}\left(\psi^{-N-n}(X), \gamma\right)\right\} \geq K_{\psi} n+B
$$

then there exists $\ell_{\infty}>0$ so that $\ell_{Q_{n}}(\gamma) \rightarrow \ell_{\infty}$. Furthermore, we have

$$
\ell_{Q_{N+n}}(\gamma) \leq 2 \ell_{Q_{N}}(\gamma)
$$

for all $n>0$.

Proof. Let $\operatorname{depth} \mathbf{t h}_{Q}(\gamma)$ denote the distance of $\mathbb{T}_{\varepsilon_{3}}^{\prime}(\gamma)$ from the boundary of the convex core of $Q$. Let $f_{2 L}$ be the function given by Corollary 7.19 so that any curve $\beta$ for which $\ell_{Q}(\beta)<2 L$ satisfies

$$
\operatorname{depth}_{Q}(\beta) \geq f_{2 L}\left(\min \left\{d_{\mathcal{C}}(X, \gamma), d_{\mathcal{C}}(\gamma, Y)\right\}\right)
$$

Let $d_{n}=\operatorname{depth}_{Q_{N+n}}(\gamma)$. If $\ell_{Q_{N+n}}(\gamma)<2 L$, then, we have

$$
d_{n} \geq f_{2 L}(K n+B) \text {. }
$$

Since $\ell_{Q_{N}}(\gamma)<L$, we know by assumption that

$$
d_{0} \geq f_{2 L}(B)
$$


Note that $f_{2 L}$ is an increasing function so we can make $f_{2 L}(B)$ as large as we like through our choice of $B$.

Let

$$
\ell_{n}=\ell_{Q_{N+n}}(\gamma) .
$$

If $\ell_{n}<2 L$ then by Theorem 5.8 there are constants $C_{1}^{\prime}$ and $C_{2}^{\prime}$ so that

$$
\left|\log \left(\frac{\ell_{n+1}}{\ell_{n}}\right)\right| \leq C_{1}^{\prime} e^{-C_{2}^{\prime} d_{n}} .
$$

Choose $C_{1}$ and $C_{2}$ such that

$$
C_{1} e^{-C_{2} n}=C_{1}^{\prime} e^{-C_{2}^{\prime} f_{2 L}\left(K_{\psi} n+B\right)}
$$

(recall $f_{2 L}$ is linear). Since $C_{1}=C_{1}^{\prime} e^{-C_{2}^{\prime} f_{2 L}(B)}$ we can choose $B$ such that

$$
\sum_{j=0}^{n} C_{1} e^{-C_{2} n} \leq \frac{C_{1}}{1-e^{-C_{2}}} \leq \log 2
$$

from which it follows that

$$
\left|\log \left(\frac{\ell_{n}}{\ell_{0}}\right)\right|<\log 2
$$

by a simple inductive argument. We conclude that $\ell_{n}<2 L$, and thus equation (8.5) holds for all $n$. It follows that $\left\{\ell_{n}\right\}$ is a Cauchy sequence, and its convergence to $\ell_{\infty}>0$ follows from (8.6).

We note the following corollary, which will play a role in establishing strong convergence of $\left\{Q_{n}\right\}$.

Corollary 8.5 If $Q_{n}$ has a subsequence that converges algebraically then for each $\gamma \in \mathcal{C}^{0}(S)$ the sequence $\ell_{Q_{n}}(\gamma)$ converges to a positive number.

Proof. Algebraic convergence of the subsequence $Q_{n_{i}}$ implies there is an $L$ so that $\ell_{Q_{n_{i}}}(\gamma)<L$ for each $n_{i}$. Taking $B$ as guaranteed by Proposition 8.4 we use Theorem 8.1 to choose subsets $U, V$ and $W$ of $\mathcal{P} \mathcal{M L}(S)$ with $\gamma \in W$ such that

$$
\min \left\{d_{\mathcal{C}}\left(\psi^{N+n}(\beta), \gamma\right), d_{\mathcal{C}}\left(\psi^{-N-n}(\alpha), \gamma\right)\right\} \geq K_{\psi} n+B
$$

for all $\alpha \in U$ and $\beta \in V$ for all $n \geq 0$. We then choose $N>0$ such that if $\psi^{N}(X) \subset V$ and $\psi^{N}(Y) \subset U$. We then have

$$
\min \left\{d_{\mathcal{C}}\left(\psi^{N+n}(Y), \gamma\right), d_{\mathcal{C}}\left(\psi^{-N-n}(X), \gamma\right)\right\} \geq K_{\psi} n+B
$$

and by Theorem 8.4 we have that $\ell_{Q_{n}}(\gamma)$ converges to a positive number. 8.5 
We now use Theorems 8.1 and 8.4 to find a pants decomposition whose lengths in $Q_{n}$ converge.

Proposition 8.6 There exists a pants decomposition $\mathcal{P}$ such that for every $\gamma \in$ $\mathcal{P}$ the sequence $\ell_{Q_{n}}(\gamma)$ converges to a positive number.

Proof. By Lemma 7.17 and the remark that follows it there is $D$-coarse path from $\psi^{-n}(X)$ to $\psi^{n}(Y)$ consisting of curves in $\mathcal{C}^{0}(S)$ whose lengths are at most $L_{g}$ in $Q\left(\psi^{-n}(X), \psi^{n}(Y)\right)$. Let $B$ be the constant given by Proposition 8.4 where $L=L_{g}^{\prime}$ is the Bers constant for a pants decomposition.

As in the proof of Corollary 8.5 we can find subsets $U, V$ and $W$ of $\mathcal{P} \mathcal{M L}(S)$ chosen with respect to the constants $B+1$ and $D$ and a positive integer $N$ such that

$$
\min \left\{d_{\mathcal{C}}\left(\psi^{N+n}(Y), \gamma\right), d_{\mathcal{C}}\left(\psi^{-N-n}(X), \gamma\right)\right\} \geq K_{\psi} n+B+1
$$

for all $n \geq 0$ and $\gamma \in W$.

In our coarse path from $\psi^{-N}(X)$ to $\psi^{N}(Y)$ consisting of curves whose length is at most $L_{g}$ in $Q_{N}$ there is a curve $\gamma \in W$. Let $f: Z \rightarrow Q_{N}$ be a 1-Lipschitz surface realizing $\gamma$. We can then extend $\gamma$ to a pants decomposition $\mathcal{P}$ on such that for all $\gamma^{\prime} \in \mathcal{P}$ we have

$$
L_{g}^{\prime}>\ell_{Z}\left(\gamma^{\prime}\right)>\ell_{Q_{N}}\left(\gamma^{\prime}\right) .
$$

Since $d_{\mathcal{C}}\left(\gamma, \gamma^{\prime}\right) \leq 1$ we have

$$
\min \left\{d_{\mathcal{C}}\left(\psi^{N+n}(Y), \gamma^{\prime}\right), d_{\mathcal{C}}\left(\psi^{-N-n}(X), \gamma^{\prime}\right)\right\} \geq K_{\psi} n+B
$$

for all $n \geq 0$. Then Theorem 8.4 implies that $\ell_{Q_{n}}\left(\gamma^{\prime}\right)$ converges for all $\gamma^{\prime} \in \mathcal{P}$.

In the following proposition we will show that we have exponential decay of the bi-Lipschitz constant on the iterated image of sufficiently deep and thick subsets. The proof has the same basic structure as the proof of Proposition 8.4

Proposition 8.7 Given $\epsilon, R, L, C>0$ there exist $B, C_{1}, C_{2}>0$ such that the following holds. Assume that $\mathcal{K}$ is a subset of $Q_{N}$ such that $\operatorname{diam}(\mathcal{K})<R$, $\operatorname{inj}_{p}(\mathcal{K})>\epsilon$ for each $p \in \mathcal{K}$ and $\gamma \in \mathcal{C}^{0}(S)$ is represented by a closed curve in $\mathcal{K}$ of length at most $L$ satisfying

$$
\min \left\{d_{\mathcal{C}}\left(\psi^{N+n}(Y), \gamma\right), d_{\mathcal{C}}\left(\psi^{-N-n}(X), \gamma\right\} \geq K_{\psi} n+B\right.
$$

for all $n \geq 0$. Then we have

$$
\log \operatorname{bilip}\left(\phi_{N+n}, p\right) \leq C_{1} e^{-C_{2} n}
$$

for $p$ in $\phi_{N+n-1} \circ \cdots \circ \phi_{N} \circ f(\mathcal{K})$ and

$$
\frac{C_{1}}{1-e^{-C_{2}}}<C
$$


Proof. As in the proof of Proposition 8.4, if $\mathcal{K}$ is a subset of $C(Q)$ we let $\operatorname{depth}_{Q}(\mathcal{K})$ be defined by the distance from $\mathcal{K}$ to $\partial C(Q)$. Let $\Phi_{n}=\phi_{N+n} \circ$ $\cdots \phi_{N}$.

Let $\epsilon_{0}=\epsilon e^{-C}$. By $\overline{\mathrm{BM}}$ there exists an $\epsilon_{1}$ such that a point within $\epsilon$ of point of injectivity radius at least $\epsilon_{0}$ will have injectivity radius at least $\epsilon_{1}$. Let $C_{1}^{\prime}$ and $C_{2}^{\prime}$ be the constants given by Theorem 5.6 for the thickness constant $\epsilon_{1}$. Let $f=f_{L e^{C}}$ be the linear function given by Corollary 7.19. We then define $d(n)=f\left(K_{\psi} n+B\right)-R-\epsilon$. Finally we choose $C_{1}$ and $C_{2}$ such that

$$
C_{1} e^{-C_{2} n}=C_{1}^{\prime} e^{-C_{2}^{\prime} d(n)} .
$$

Note that $C_{1}=C_{1}^{\prime} e^{-C_{2}^{\prime} d(0)}$ and we can make $d(0)$ as large as we like through our choice of $B$. Therefore we can choose $B$ such that

$$
\frac{C_{1}}{1-e^{-C_{2}}}<C \text {. }
$$

With this setup it is now easy to complete the proof of the theorem via induction. Note that if $p \in \mathcal{K}$ then we have $\operatorname{depth}_{Q_{N}}\left(B_{\epsilon}(p)\right)>d(0)$ where $B_{\epsilon}(p)$ is the ball of radius $\epsilon$ centered at $p$. By Theorem 5.6 for all $q \in B_{\epsilon}(p)$ we have

$$
\log \operatorname{bilip}\left(\phi_{N}, q\right)<C_{1} .
$$

In particular for every $p \in \mathcal{K}$ we have

$$
\log \operatorname{bilip}\left(\phi_{N}, p\right)<C_{1}
$$

and every point in $\phi_{N}(\mathcal{K})$ has injectivity radius at least $\epsilon e^{-C_{1}}$.

Let

$$
c_{n}=\sum_{i=0}^{n} C_{1} e^{-C_{2} i}
$$

and note that

$$
c_{n}<\frac{C_{1}}{1-e^{-C_{2}}}<C .
$$

Assume that the theorem holds for all $i$ between 0 and $n$ and that the injectivity radius of every point in $\Phi_{i}(\mathcal{K})$ is greater than $\epsilon e^{-c_{i}}>\epsilon e^{-C}$. Note that if $p$ is in $\mathcal{K}$ then

$$
\log \operatorname{bilip}\left(\Phi_{n}, p\right)<c_{n}<C \text {. }
$$

It follows that the length of $\Phi_{n}(\gamma)$ is $<2 L_{g} e^{C}$ and for every point $p$ within $\epsilon$ of $\Phi_{n}(\mathcal{K})$ we have $\operatorname{depth}_{Q_{N+n}}(p)>d(n)$. We also note that the injectivity radius at $p$ will be greater than $\epsilon_{1}$ so we can apply Theorem [5.6 to see that

$$
\log \operatorname{bilip}\left(\phi_{N+n+1}, p\right)<C_{1} e^{-C_{2}(n+1)}
$$

and that at every point in $\phi_{N+n+1} \circ \Phi_{n}(\mathcal{K})=\Phi_{n+1}(\mathcal{K})$ the injectivity radius is at least $\epsilon^{-c_{n+1}}$. This completes the proof of the induction hypothesis and the proposition. 
Proposition 8.8 There exists a positive integer $N$, positive constants $C_{1}, C_{2}$ and a 1-Lipschitz homotopy equivalence $f: Z \rightarrow Q_{N}$ so that for all points $p$ in $\phi_{N+n-1} \circ \cdots \phi_{N} \circ f(Z)$ we have

$$
\log \operatorname{bilip}\left(\phi_{N+n}, p\right)<C_{1} e^{-C_{2} n} .
$$

Proof. By Proposition 8.6 there exists a pants decomposition $\mathcal{P}$ such that $\ell_{Q_{n}}(\gamma)$ converges to a positive number for every $\gamma \in \mathcal{P}$. In particular there are constants $L^{+}>L^{-}>0$ such that $L^{+}>\ell_{Q_{n}}(\gamma)>L^{-}$for all $n$ and $\gamma \in \mathcal{P}$. For each $n$ let $f_{n}: Z_{n} \rightarrow Q_{n}$ be a 1-Lipschtiz homotopy equivalence realizing $\mathcal{P}$. By the collar lemma, there exists $\epsilon>0$ such that any hyperbolic surface with a pants decomposition whose lengths are between $L^{-}$and $L^{+}$is $\epsilon$-thick. In particular the surfaces $Z_{n}$ are $\epsilon$-thick. We also note that there is an $R>0$ such that an $\epsilon$-thick surface has diameter bounded above by $R$.

By Lemma 7.8 there exists an $\epsilon^{\prime}>0$ such that $f_{n}\left(Z_{n}\right)$ is contained in the $\epsilon^{\prime}$-thick part of $Q_{n}$. Let $B$ be the constant given by Proposition 8.7 for the constants $\epsilon^{\prime}, R, L^{+}$and $C=2$. (Note that the choice of 2 is completely arbitrary and could be any number $>1$ ). Using Theorem 8.1 we can find an integer $N$ such that

$$
\min \left\{d_{\mathcal{C}}\left(\psi^{N+n}(Y), \gamma\right), d_{\mathcal{C}}\left(\psi^{-N-n}(X), \gamma\right\} \geq K_{\psi} n+B\right.
$$

where $\gamma$ is a curve in $\mathcal{P}$. When then let $f=f_{N}$ and $Z=Z_{N}$ and proposition follows from Proposition 8.7

We are now ready to prove the convergence of double iteration, Theorem 8.3

Proof (Proof of Theorem 8.3). Let $f: Z \rightarrow Q_{N}$ be the 1-Lipschtiz surface given by Proposition 8.8. Then the maps $f_{n}=\phi_{N+n-1} \circ \cdots \phi_{N} \circ f$ are $C$-Lipschitz where

$$
C=\frac{C_{1}}{1-e^{-C_{2}}} .
$$

By Proposition 7.9 the sequence $\left\{\left(f_{n}, Q_{n}\right)\right\}$ has a convergent subsequence $\left\{\left(f_{n_{i}}, Q_{n_{i}}\right)\right\}$ in $A H(S)=A H(Z)$. Let $\left\{\left(f_{\infty}, Q_{\infty}\right)\right\}$ be the limit. Note that from the proof of Proposition 7.9 we can assume that there are lifts $\tilde{f}_{n_{i}}$ that converge to $\tilde{f}_{\infty}$.

Since Corollary 8.5 guarantees the limit has no parabolics, Theorem 7.5 implies that the limit is strong. In particular if we pick a point $p$ in $Z$ and let $p_{n}=f_{n}(p)$ then the sequence $\left\{\left(Q_{n_{i}}, p_{n_{i}}\right)\right\}$ will converge geometrically to $\left(Q_{\infty}, p_{\infty}\right)$ for some point $p_{\infty} \in Q_{\infty}$. Furthermore if $\mathcal{K}$ is a compact set with $f_{\infty}(S) \subset \mathcal{K}$ and $g_{n_{i}}:\left(\mathcal{K}, p_{\infty}\right) \rightarrow\left(Q_{n_{i}}, p_{n_{i}}\right)$ are approximating maps then $f_{n_{i}}$ is homotopic to $g_{n_{i}} \circ f_{\infty}$.

We will show that the entire sequence $\left\{\left(Q_{n}, p_{n}\right)\right\}$ converges geometrically to $\left(Q_{\infty}, p_{\infty}\right)$. Let $\gamma \in \mathcal{C}^{0}(S)$ be a simple closed curve on $S$ and represent it by a closed curve $\gamma_{\infty}$ in $M_{\infty}$ and let $L=\ell_{M_{\infty}}\left(\gamma_{\infty}\right)$. Let $\mathcal{K}$ be a compact set in $M_{\infty}$ and assume that both $p_{\infty}$ and $\gamma_{\infty}$ is contained in $\mathcal{K}$. To show geometric 
convergence we need to show that for any $A>0$ there exists $e^{A}$-bi-Lipschitz embeddings

$$
g_{n}:\left(\mathcal{K}, p_{\infty}\right) \rightarrow\left(Q_{n}, p_{n}\right)
$$

for $n$ sufficiently large.

Let $B, C_{1}$ and $C_{2}$ be the constants given by Proposition 8.7 with repsect to the constants $\epsilon e^{-A / 2}, R e^{A / 2}, L e^{A / 2}$ and $A / 2$. By Theorem 8.1 there exists an $N_{A}$ such that

$$
\min \left\{d_{\mathcal{C}}\left(\psi^{N_{A}+n}(Y), \gamma\right), d_{\mathcal{C}}\left(\psi^{-N_{A}-n}(X), \gamma\right\} \geq K_{\psi} n+B .\right.
$$

Let $\mathcal{K}^{\prime}$ be the closed $\epsilon$-neighborhood of $\mathcal{K}$. By the strong convergence of the subsequence for $n_{i}$ sufficiently large there is a $e^{A / 2}$-bi-Lipschitz embedding

$$
g_{n_{i}}:\left(\mathcal{K}^{\prime}, p_{\infty}\right) \rightarrow\left(M_{n_{i}}, p_{n_{i}}\right) .
$$

Note that every point in $g_{n_{i}}(\mathcal{K})$ will have injectivity radius at least $\epsilon e^{-A / 2}$, the diameter of $g_{n_{i}}(\mathcal{K})$ will be at most $R e^{A / 2}$ and the length of $g_{n_{i}}\left(\gamma_{\infty}\right)$ will be at most $L e^{A / 2}$. Since we can always replace $N_{A}$ with a larger integer we can assume $N_{A}=n_{i}$ where $n_{i}$ is part of the convergent subsequence. We now apply Theorem 8.7 to $g_{N_{A}}(\mathcal{K})$ which implies that $\phi_{N_{A}+n-1} \circ \cdots \circ \phi_{N_{A}}$ is $e^{A / 2}$ bi-Lipschitz on $g_{N_{A}}(\mathcal{K})$. Therefore the composition

$$
g_{N_{A}+n}=\phi_{N_{A}+n-1} \circ \cdots \circ \phi_{N_{A}} \circ g_{N_{A}}
$$

is $e^{A}$-bi-Lipschitz. Furthermore $g_{n}\left(p_{\infty}\right)=p_{n}$ so we have our desired bi-Lipschitz embeddings and $\left\{\left(Q_{n}, p_{n}\right)\right\}$ converges geometrically to $\left(M_{\infty}, p_{\infty}\right)$.

To see that the sequence also converges algebraically assume that $\mathcal{K}$ contains $f_{\infty}(S)$. We note that $f_{n+1}$ is homotopic to $\phi_{n} \circ f_{n}$ and more generally $f_{n+k}$ is homotopic to $\phi_{n+k} \circ \cdots \circ \phi_{n} \circ f_{n}$. On the subsequence $\left\{n_{i}\right\}$ we already know that $g_{n_{i}} \circ f_{\infty}$ is homotopic to $f_{n_{i}}$. By the above fact, the composition $\phi_{N_{A}+n-1} \circ \cdots \circ \phi_{N_{A}} \circ f_{N_{A}}$ is homotopic to $f_{N_{A}+n-1}$ and in turn homotopic to $g_{N_{A}+n-1} \circ f_{\infty}$. If $g_{n}$ are approximating maps for $\mathcal{K}$ whose bi-Lipschitz constant limits to 1 then by Lemma 7.4 we have that $\left(g_{n} \circ f_{\infty}, Q_{n}\right) \rightarrow\left(f_{\infty}, M_{\infty}\right)$. By the above remarks $\left(g_{n} \circ f_{\infty}, Q_{n}\right) \in\left[\left(f_{n}, Q_{n}\right)\right]$ and therefore $\left[\left(f_{n}, Q_{n}\right)\right]$ converges to $\left[\left(f_{\infty}, M_{\infty}\right)\right]$ algebraically.

We conclude with the proof of Theorem 1.3 ,

Theorem 1.3 (Mapping Torus Hyperbolic) Let $\psi \in \operatorname{Mod}(S)$ be pseudoAnosov. Then the mapping torus $T_{\psi}=S \times[0,1] /(x, 0) \sim(\psi(x), 1)$ admits a complete hyperbolic structure.

Proof. We note that as $\operatorname{Mod}(S)$ acts diagonally on quasi-Fuchsian space by re-marking, the manifolds $Q_{n}$ and $\psi\left(Q_{n}\right)$ are isometric. Because we have

$d\left(\psi^{-n+1}(X), \psi^{-n}(X)\right)=d(\psi(X), X) \quad$ and $\quad d\left(\psi^{n+1}(Y), \psi^{n}(Y)\right)=d(\psi(Y), Y)$, 
and

$$
\psi\left(Q_{n}\right)=Q\left(\psi^{-n+1}(X), \psi^{n+1}(Y)\right),
$$

there is a uniform $K$ for which $Q_{n}$ admits a $K$-bi-Lipschitz self-diffeomorphism

$$
\Psi_{n}: Q_{n} \rightarrow Q_{n}
$$

so that $\Psi_{n}$ is in the homotopy class of $\psi$.

We now use inflexibility and the fact that $\left(Q_{n}, p_{n}\right)$ converges geometrically to $\left(Q_{\infty}, p_{\infty}\right)$ to extract a limiting isometry

$$
\Psi_{\infty}: Q_{\infty} \longrightarrow Q_{\infty}
$$

in the homotopy class of $\psi$ as a limit directly.

If $\mathcal{K}$ is a compact set in $Q_{\infty}$ containing $p_{\infty}$ with geometric limit mappings $g_{n}:\left(\mathcal{K}, p_{\infty}\right) \longrightarrow\left(Q_{n}, p_{n}\right)$, then $\left(g_{n}\right)^{-1} \circ \Psi_{n} \circ g_{n}$ converges up to subsequence to a uniformly bi-Lipschitz $\Psi_{\infty}$ where the conjugating maps are defined. Observe that since $\operatorname{depth}_{Q_{n}}\left(p_{n}\right) \rightarrow \infty$ as $n \rightarrow \infty$, the compact sets $g_{n}(\mathcal{K})$ are arbitrarily deep in the convex core of $Q_{n}$ as $n \rightarrow \infty$. Theorem [5.6 then implies that for any $\epsilon>0$ and any $\mathcal{K}$ the maps $\Psi_{n}$ can be taken to be $(1+\epsilon)$-bi-Lipschitz on $g_{n}(\mathcal{K})$ for $n$ sufficiently large. Diagonalizing, the limit $\Psi_{\infty}$ is an isometry. As the group of isometries of any hyperbolic 3 -manifold is discrete, we may pass to the quotient by the action of $\left\langle\Psi_{\infty}\right\rangle$. The quotient of $Q_{\infty} /\left\langle\Psi_{\infty}\right\rangle$ is a hyperbolic 3 -manifold with the fundamental group $\pi_{1}\left(T_{\psi}\right)$, and is thus homeomorphic $T_{\psi}$ by Stallings' Theorem [St].

Remark. Note that in Thurston's original proof significant extra work is required to show that the limit $Q_{\infty}$ is doubly degenerate, or that the limit set of $\Gamma_{\infty}$ is the entire sphere (see [Ot, Sec. 6.2]). In our approach the double degeneracy of $Q_{\infty}$ is immediate from our estimates on the depth of the basepoint in the convex core and the strong convergence of $Q_{n}$.

\section{References}

[Ag] I. Agol. Tameness of hyperbolic 3-manifolds. Preprint, arXiv: mathGT/0405568 (2004).

[Ah] L. Ahlfors. Invariant operators and integral representations in hyperbolic space. Math. Scan. 36(1975), 27-43.

[And] M. Anderson. The Dirichlet problem at infinity for manifolds of negative curvature. J. Diff. Geom. 18(1983), 701-721.

[Brs1] L. Bers. Simultaneous uniformization. Bull. AMS 66(1960), 94-97.

[Brs2] L. Bers. On boundaries of Teichmüller spaces and on kleinian groups: I. Annals of Math. 91(1970), 570-600. 
[BeFu] M. Bestvina and K. Fujiwara. Bounded cohomology of subgroups of mapping class groups. Geometry and Topology 6(2002), 69-89.

[Bon1] F. Bonahon. Bouts des variétés hyperboliques de dimension 3. Annals of Math. 124(1986), 71-158.

[Bon2] F. Bonahon. The geometry of Teichmüller space via geodesic currents. Invent. math. 92(1988), 139-162.

[Bow] B. Bowditch. Some results on the geometry of convex hulls in manifolds of pinched negative curvature. Comment. Math. Helv. 69(1994), 49-81.

[BH] M. Bridson and A. Haefliger. Metric Spaces of Non-Positive Curvature. Springer-Verlag, 1999.

[BB] J. Brock and K. Bromberg. On the density of geometrically finite Kleinian groups. Acta Math. 192(2004), 33-93.

[BBES] J. Brock, K. Bromberg, R. Evans, and J. Souto. Maximal cusps, ending laminations and the classification of Kleinian groups. In preparation (2008).

$[\mathrm{BCM}]$ J. Brock, R. Canary, and Y. Minsky. The classification of Kleinian surface groups, II: the ending lamination conjecture. Submitted (2004).

[Brm1] K. Bromberg. Hyperbolic cone manifolds, short geodesics, and Schwarzian derivatives. J. Amer. Math. Soc. 17(2004), 783-826.

[Brm2] K. Bromberg. Projective structures with degenerate holonomy and the Bers density conjecture. Annals of Math. 166(2007), 77-93.

[BS] K. Bromberg and J. Souto. Density of Kleinian groups. In preparation.

[BM] R. Brooks and J. P. Matelski. Collars for Kleinian Groups. Duke Math. J. 49(1982), 163-182.

[Bus] P. Buser. Geometry and Spectra of Compact Riemann Surfaces. Birkhauser Boston, 1992.

[CG] D. Calegari and D. Gabai. Shrinkwrapping and the taming of hyperbolic 3-manifolds. J. AMS 19(2006), 385-446.

[Can] R. D. Canary. Ends of hyperbolic 3-manifolds. J. Amer. Math. Soc. 6(1993), 1-35.

[CCHS] R. D. Canary, M. Culler, S. Hersonsky, and P. B. Shalen. Approximation by maximal cusps in the boundaries of quasiconformal deformation spaces. J. Diff. Geom. 64(2003), 57-109.

$[\mathrm{CH}] \quad$ R. D. Canary and S. Hersonsky. Ubiquity of geometric finiteness in boundaries of deformation spaces of hyperbolic 3-manifolds. Amer. J. of Math. 126(2004), 1193-1220. 
[CT] J. W. Cannon and W. P. Thurston. Group invariant Peano curves. Geometry and Topology 11(2007), 1315-1355.

[Dum] D. Dumas. Complex projective structures. In Handbook of Teichmüller Theory, Volume II. EMS Publishing House, 2008.

[Ev] R. Evans. Tameness persists in weakly type-preserving strong limits. Amer. J. Math. 126(2004), 713-737.

[FLP] A. Fathi, F. Laudenbach, and V. Poénaru. Travaux de Thurston sur les surfaces, volume 66-67. Astérisque, 1979.

[HK] C. Hodgson and S. Kerckhoff. Rigidity of hyperbolic cone-manifolds and hyperbolic Dehn surgery. J. Diff. Geom. 48(1998), 1-59.

[IT] Y. Imayoshi and M. Taniguchi. An Introduction to Teichmüller Spaces. Springer-Verlag, 1992.

[MM] H. Masur and Y. Minsky. Geometry of the complex of curves I: hyperbolicity. Invent. Math. 138(1999), 103-149.

[Mc1] C. McMullen. Iteration on Teichmüller space. Invent. math. 99(1990), 425-454.

[Mc2] C. McMullen. Cusps are dense. Annals of Math. 133(1991), 217-247.

[Mc3] C. McMullen. Renormalization and 3-Manifolds Which Fiber Over the Circle. Annals of Math. Studies 142, Princeton University Press, 1996.

[Min1] Y. Minsky. The classification of punctured torus groups. Annals of Math. 149(1999), 559-626.

[Min2] Y. Minsky. The classification of Kleinian surface groups I: models and bounds. Annals of Math. 171(2010), 1-107.

[Mum] D. Mumford. A remark on Mahler's compactness theorem. Proc. AMS 28(1971), 289-294.

[Neh] Z. Nehari. Schwarzian derivatives and schlicht functions. Bull. AMS 55(1949), 545-551.

[Ot] J. P. Otal. Le théorème d'hyperbolisation pour les variétés fibrées de dimension trois. Astérisque, 1996.

[Rei] H.M. Reimann. Invariant extension of quasiconformal deformations. Ann. Acad. Sci. Fen. 10(1985), 477-492.

[St] J. Stallings. On fibering certain 3-manifolds. In Topology of 3manifolds, pages 95-100. Prentice Hall, 1962. 
[Sul] D. Sullivan. On the ergodic theory at infinity of an arbitrary discrete group of hyperbolic motions. In Riemann Surfaces and Related Topics: Proceedings of the 1978 Stony Brook Conference. Annals of Math. Studies 97, Princeton, 1981.

[Th1] W. P. Thurston. Geometry and Topology of Three-Manifolds. Princeton lecture notes, 1979.

[Th2] W. P. Thurston. Hyperbolic structures on 3-manifolds II: Surface groups and 3-manifolds which fiber over the circle. Preprint, arXiv:math.GT/9801045 (1986).

Brown University

UNIVERSITY OF UTAH 